\title{
High-temperature superconductivity: From macro- to nanoscale structures
}

\author{
A. N. Kovalenko \\ Ioffe Institute, St. Petersburg, Russia \\ ras-kan@mail.ru
}

DOI 10.17586/2220-8054-2016-7-6-941-970

\begin{abstract}
The analysis of achievements, problems and prospects of high-temperature superconductivity (HTSC) in the macro- and nanostructured materials has been given. The main experimental results and theoretical models describing the physical mechanisms of the superconductivity appearance at phenomenological and microscopic levels, including change in the energy spectrum of atoms in these materials with the advent of the 'superconducting' gap at temperatures below the critical transition, as well as the above-critical temperature 'pseudo-gap' have been analyzed. Although the origin of the pseudo-gap is not completely understood, it can be considered as an independent phase transition in the substance prior to the transition to the zero resistance state and insusceptibility (or insensitivity) to external magnetic field in high-temperature superconductors. Features of multi-gap and gapless superconducting materials as well as their ability to further increase the temperature of supercritical transition are discussed. Large resources to create the necessary electron and phonon spectra in the process of high-temperature superconductivity formation are associated with the use of nanoscale structures and nanoparticles of conductors and dielectrics. Electrical conductive contacts between nanoparticles and tunnel chains of nanoclusters, where delocalized electron spectra form similar energy shells to the atomic or nuclear shells, play a significant part here. It is required to further investigate the occurrence of high-temperature superconductivity at the level of interphase layers (non-autonomous phases) in nanostructures containing a large fraction of the substance in this condition. It is essential to develop adequate methods for synthesis of nanoparticles of variable size, structure and morphology, as well as techniques for their consolidation that would ensure the preservation of superconductivity of individual nanoparticles, their chemical, thermal, magnetic and current stability in the dissipative processes with functional and fluctuation effects.
\end{abstract}

Keywords: high-temperature superconductivity, energy spectrum, 'superconducting' and 'pseudo-' gaps, layered and nanoscopic structures, non-autonomous phases, chemical, thermal, current, and magnetic resistance.

Received: 20 August 2016

Revised: 27 October 2016

\section{Contents}

1. Introduction

2. Superconductivity thermodynamics and electrodynamics

3. Energy spectrum of superconducting materials, energy 'gap' and 'pseudo-gap' in HTSC

4. Energy 'pseudo-gap' as an independent phase transition

5. Peculiar properties of nanostructure superconductivity

6. Dissipative phenomena. Chemical, thermal, current and magnetic resistance of nanostructured HTSC materials

7. Conclusion

8. Post Scriptum

Acknowledgements

References

\section{Introduction}

The phenomenon of superconductivity occurs in certain substances and materials at temperatures below a critical value, $T c$, which is specific for each material. Phenomenologically, it is considered as the combined effect of the complete absence of electrical DC resistance and negligible susceptibility to an external magnetic field [1].

The electric effect of superconductivity was discovered by H. Kamerlingh-Onnes in 1911 by means of mercury experiments at very low temperatures. He found that the electrical resistance of mercury gradually decreases with reducing temperature to $\sim 4.2 \mathrm{~K}$ and then almost abruptly becomes zero [2]. Thereafter, a similar phenomenon was observed for a number of other metals (lead, tin, thallium, uranium, etc.) when cooled to temperatures close to absolute zero. It was also accompanied by changes in their thermal properties in contrast to behavior of metals that cannot be superconductive, such as silver, copper, gold, platinum, etc. In the latter case, the electrical resistance gradually decreased with temperature, approaching a residual (zero if a metal is hypothetically pure) value at $0 \mathrm{~K}$. 
Magnetic effect of superconductivity was discovered by W. Meissner and R. Ochsenfeld in 1933. They demonstrated that in parallel, the superconductors are technically perfect diamagnetic materials if they are in a magnetic field of a magnitude less than a critical value $H c$, i.e. they push magnetic lines completely out of the sample volume [3,4]. The description of this effect in terms of electrodynamics, proposed in 1935 by brothers F. London and H. London [5] in addition to J.C. Maxwell's [6] equations, allowed us to estimate the depth of magnetic field penetration into the superconductor. In the case of metals, this area covers hundreds of atomic layers and has the skin layer of superconducting current of thickness $\sim 10-100 \mathrm{~nm}$. The proposed approach also allowed us to associate the superconductivity with the transition of current carriers to the state with the least possible momentum.

The phenomenological theory of superconductivity [7] created by V. L. Ginzburg and L. D. Landau in 1950 describes the transition to the superconducting state as the formation of phase coherence in the substance electronic system but does not determine the charge of the superconducting current carriers and does not explain the causes of superconductivity. The Ginsburg-Landau equations derived on this basis involving a L. P. Gorkov's microscopic approach [8] in 1958, match well with the superconductor behavior at critical temperatures and are still used for the experimental data interpretation and in technical applications [9]. The detailed microscopic theory of superconductivity [10] was developed in 1957 by J. Bardeen, L. N. Cooper and J. R. Schrieffer. It named as the BCS-theory - using the first letters of their surnames. According to this theory, electrons experience attraction at temperatures below $T c$ due to the interaction with the thermal vibrations of positively charged crystal lattice. Their excitations, in the form of acoustic frequency elastic waves and corresponding quasi-particles (phonons), are distributed over the entire space of the material. In the context of quantum mechanics, it is described as the electron-phonon interaction that does not stop, even at absolute zero, according to Heisenberg's uncertainty principle. Due to the fact that it is stronger than the Coulomb repulsion between the electrons, some electrons (with energies close to the Fermi level) are merged into so-called Cooper pairs. According to the Pauli exclusion principle, in contrast to individual initial states of electrons as fermion particles with half-integer spin which comply with Fermi-Dirac statistics, the paired electrons behave coherently as a single quantum-mechanical system of identical boson particles with integer spin which comply with the Bose-Einstein statistics [11]. Due to this fact, they cannot exchange energy portions with the crystal lattice that are lower than the energy resulting from their coupling. Since, according to the principle of wave-particle duality, any particle can be viewed as a wave of certain amplitude, frequency and phase according to de Broglie relations [12], Cooper pairs as coherent quasiparticles constitute a single wave together. It is described with the general wave function where the amplitude square determines the density of Cooper pairs on the spatial scale (length) of coherence. Herewith, there is no scattering of electron waves on thermal vibrations of the substance lattice and the paired conduction electrons can flow through it without energy loss and therefore without resistance, which also does not allow the external magnetic field to penetrate inside the material. To meet these requirements for conventional materials, according to the classical Bardeen-Cooper-Schrieffer theory, the critical temperature may not exceed the limit of about $40 \mathrm{~K}$ $\left(-233^{\circ} \mathrm{C}\right)$.

It should be noted that it was G. Fröhlich who first identified the connection of the superconductivity phenomenon with the lattice vibrations in 1950 . He proposed a hypothesis of the influence of the ion mass on the critical transition temperature, with the latter being inversely proportional to the square root of the ionic mass in the isotope collection of the given superconductor [13]. Experiments with isotopic substitutions conducted by E. Maxwell and other researchers [14,15], confirmed the isotopic effect predicted by G. Fröhlich and showed that there is a direct effect of ion mass on inter-ion distances in the lattice and the value of the Fermi energy. Consequently, the existence of the isotopic effect cannot be a reliable proof of the phonon mechanism as being solely responsible for the pairing of electrons and the appearance of superconductivity. The BCS theory is also found insufficient in terms of its inability to explain why particular superconductors have a certain critical temperature and therefore in terms of impossibility to predict the ways to increase it. This has led to the creation of other high-temperature superconductivity models (currently, there are more than 100 proposed) where various mechanisms of electrons pairing are considered [16]. They use ideas about spin and charge fluctuations, polarons, plasmons, excitons, magnetons, magnons, solitons and other quasi-particles acting as energy quanta of excitation waves in the system, as well as about the super-exchange between them and the electrons which complements electron-phonon or direct interaction between the electrons. Other ideas are expressed as well, even those concerning the opposition of superconductivity to 'super-magnetism' [17]. However, these developments have neither resulted in noticeable progress in order to understand the superconductivity phenomenon nor made it possible to predict the composition and structure of new superconductors. An indicative opinion in this respect is the one of B. T. Matthias who managed to synthesize, apparently, the largest number of new superconductors [18]. He argued that, despite the existence of numerous theories of superconductivity, the only concept that helped him in the discovery of new 
superconductors was D. I. Mendeleev's periodic table of elements. This situation has been persisting in the theory of superconductivity so far. The developed theories do not explain the possibility of creating high-temperature superconducting materials (HTSC) which are studied mostly empirically, by complication of their composition and structure and identifying correlations between the obtained properties, occurring physical and chemical processes and external thermal, electrical, magnetic and other influences. Fundamental and practical physical and chemical problems and results of these studies are published in numerous original articles as well as in various summary reviews and monographs [19-36] where other details and additional references can be found.

This review deals with the poorly represented but promising and intensively developing directions of modern research in the field of high-temperature superconductivity nanostructures. In addition, major statements of the above papers which are important for the analysis of the subject are also addressed as appropriate in the review.

\section{Superconductivity thermodynamics and electrodynamics}

Thermodynamically, the transition to the superconducting state is associated with the substance phase changes (phase transitions) [37] occurring when the temperature changes and accompanied by the changes in some of its thermodynamic and electromagnetic properties. According to the general thermodynamic theory, the transition to superconductivity and vice versa can be first and second order, which is confirmed experimentally (Fig. 1).

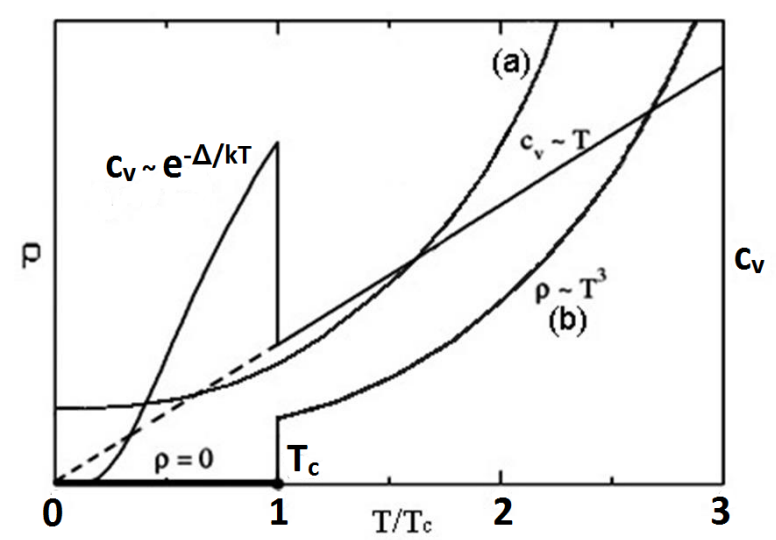

FIG. 1. The pattern of electrical resistance decrease $(\rho)$ and heat capacity variations (CV) for substances that do not become (a) and become (b) superconductive when cooled (taken from [70])

The first order transition is characterized by an abrupt change of extensive parameters at the $T c$ transition point, above all, of thermal energy, entropy and volume which are the first derivatives of the thermodynamic potential of the system on its intensive parameters, temperature and pressure. The second order transition is characterized by an abrupt change in the second derivatives of the thermodynamic potential (thermal capacity, thermal expansion factor, different ways of responsiveness) while the internal energy, density and other first order derivatives, according to the parameter set of the general system condition, are maintained at the transition point. Thermodynamic fluctuations arising at the superconducting phase emerge at the spatial scale, corresponding to the coherence length. Phase symmetry can change simultaneously as well; however, phase transitions in superconductors are not caused by changing the crystalline state of the body and the lattice symmetry and are only connected with change of its qualitative electromagnetic properties [38].

The idea of two existing superconductor orders which differ in response to the external magnetic field was first proposed in 1952 by A. A. Abrikosov and N. I. Zavaritsky $[39,40]$ based on experimental results obtained by L. B. Schubnikov for magnetization curves of superconducting alloys [41,42], and data obtained by N. I. Zavaritsky for the critical magnetic fields of thin superconducting films [43]. The soft superconductor transition from a normal to a superconducting state, depending on external magnetic conditions, can be both first and second order phase transition, whereas the hard superconductor transition from a normal to a superconducting state shall be a second order phase transition only under all conditions. Herewith, soft superconductors have the only $H c$ value of the critical magnetic field intensity; they are not superconductive if it increases. However, the possible distortions of the field due to the heterogeneous interaction with the differently-shaped conductor segments can result in the simultaneous existence of large alternating areas of superconducting and normal phases separated by a substance in the 'intermediate' phase in this sample. The hard superconductors have two critical magnetic field $H c 1$ and $H c 2$ 
values, between which it partially penetrates into the superconductor in the form of so-called Abrikosov vortex lattice [40] whose cores are non-superconducting (normal), and the superconducting currents screening the normal vortex area circulate around them. As a result, $\mathrm{Hcl}$ field transforms the conductor into the 'mixed' (metastable in nature) state and preserves its superconducting properties, and the field above $H c 2$ destroys them. Experimental observation of the structures of soft and hard superconductors being in the intermediate and mixed states was carried out in 1967 by H. Tauble and U. Essman [44], and N. V. Sarma [45, 46] using the electron microscope of $10 \mathrm{~nm}$ resolution. The schematic figure of magnetic field distribution in the conductors of different types is represented in Fig. 2 according to [31,38].

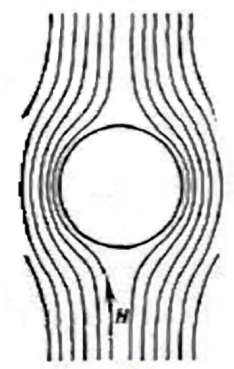

a)

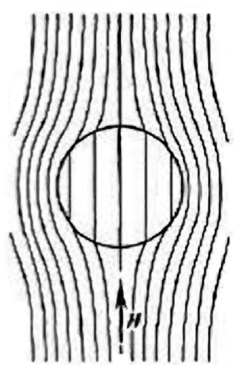

b)

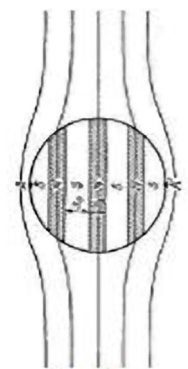

c)

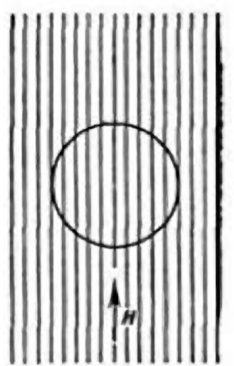

d)

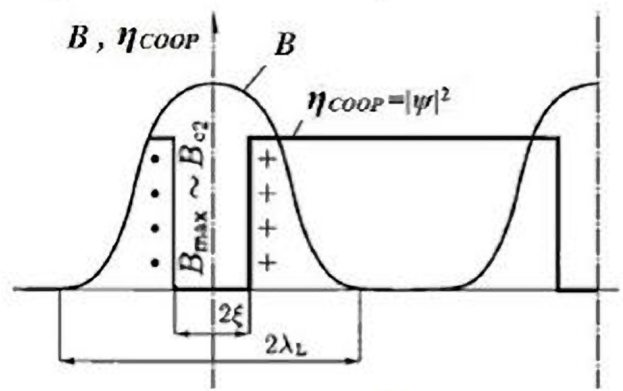

e)

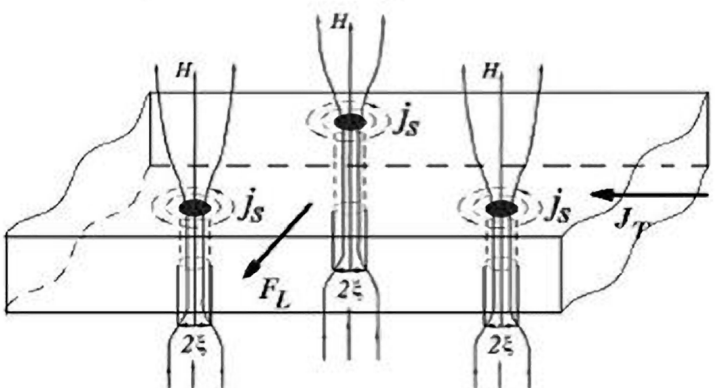

f)

FIG. 2. Magnetic field distribution around the superconducting body (Figures taken from [31, 38]): a) - superconducting state; b) and c) - intermediate state where the alternating superconducting and normal areas emerge; d) - normal state; e) - hypothetical structure of the superconducting vortex (quantum filaments of magnetic flux, where crosses and dots show schematically the direction of a superconducting vortex current); $\mathrm{f}$ ) - the Schubnikov phase (diagram); $\psi$-amplitude of the common wave function of the coherent Cooper pairs, where the amplitude square determines the $\eta$-density of Cooper pairs; e) spatial pattern of the mixed state (the specific section is shown, where $H=B / \mu_{0}$ is for the magnetic field tension and induction, $j_{S}$ stands for screening supercurrent, $2 \xi$ stands for the vortex core diameter, $J_{T}$ stands for the transport current, $F_{L}$ stands for the Lorentz force)

Along with the 'diamagnetic' scenario of superconductivity destruction, the alternative, 'paramagnetic', is possible where the strong magnetic field can change the spin orientation of one of the electrons in a Cooper pair and simultaneously change its energy similar to the known P. Zeeman splitting effect [47]. As a result, the pair changes into an unstable triplet and superconductivity is destroyed. In conventional superconductors, the 'diamagnetic' mechanism is actuated prior to the 'paramagnetic' one. However, in iron-based superconductors where the 'paramagnetic' mechanism is more effective, the preservation of superconductivity is possible even when it is not supposed to exist with respect to this mechanism. Theoretically, such an effect was predicted almost simultaneously by R. Ferrell, P. Fulde [48] and A. I. Larkin, Yu. N. Ovchinnikov [49]. According to this, when the spin orientation of one of the electrons in a Cooper pair is changed by the magnetic field along with a simultaneous change of its energy, the Fermi energy of this pair will also change, resulting in a difference from the Fermi energy of unchanged pairs, and as a consequence, the superconductivity will be preserved. The new phase of the superconducting electron liquid, predicted by them, was called the FFLO phase using the first letter of their surnames [50]. 
As confirmed by numerous experiments, superconductors can change from one type to another. Impurities in the materials play a fundamental role in such transitions [38]. The theory of such 'impure' superconductors was suggested by P.W. Anderson in 1959 [51]. There is also a theoretical background for discussion and some experimental evidence for the existence of type 1.5 superconductors which comprise the combined properties of type 1 and 2 [52,53]. According to theoretical considerations [52] when a magnetic field increases, type 1.5 superconductors are characterized by a coherent transition from Meissner 'vortex-free' condition with the complete expulsion of magnetic field lines from the sample volume in weak fields to a certain intermediate state. Here, vortices penetrate into the superconductor not forming a regular triangular lattice of Abrikosov vortices repelling one other as in type 2 superconductors but some unstable irregular clusters of vortices which attract to each other as during the transition to the normal area of type 1superconductors. Upon further magnetic field amplification, they change to a mixed or vortex state of type 2 superconductor where the Abrikosov homogeneous triangular lattice is formed. This is reminiscent of the behavior of molecular forces repulsing vortices from each other at short distances and attracting at long distances whereby vortex combinations resulting in the latter case were called by authors of the theory as vortex 'molecules'. Experiments with single crystals of magnesium diboride, $\mathrm{MgB}_{2}$, [53] revealed that the magnetic field really penetrates into them as it was theoretically predicted, that is, when a magnetic field $H<H c 1$ is weak, the superconductor is in Meissner condition and if the field amplifies, the vortices penetrate into the superconductor and form a web-shaped lattice instead of a triangular one (Fig. 3a). Further gradual magnetic field amplification leads to transformation of the web-shaped vortex lattice into alternating stripes and the formation of vortex clusters of differing density (Fig. 3b) approximately $\sim 100 \mathrm{~nm}$ in size and containing about $30-50$ vortices, and then the regular triangular lattice of Abrikosov vortices as in type 2 superconductors will appear (Fig. 3c).
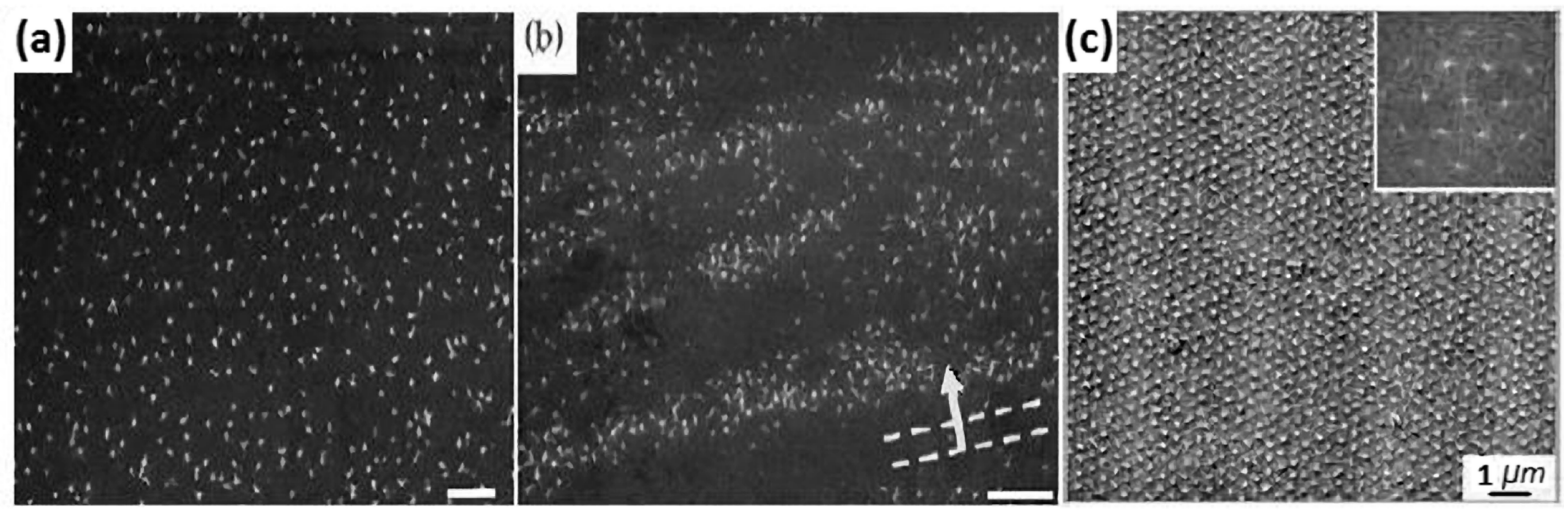

FIG. 3. Web-shaped (a, b) and regular (c) vortex structures in $\mathrm{MgB}_{2}$ films: a) at $4.2 \mathrm{~K}$ in an external magnetic field with induction of $0.0001 \mathrm{~T}$ and $\mathrm{b}$ ) at the same temperature, but in the field of $0.0005 T$ (Figure taken from the article [53]), c) at a temperature of $T \sim 6 \mathrm{~K}$ and in a weak magnetic field (Figure taken from the article [54])

However, those with differing opinions will point out that the formation of irregular vortex lattice and its flow in weak fields corresponds to the well-known phenomenon of pinning - the magnetic field $H>H c 1$ penetration into a type 2 superconductor in the form of separate ('quantized') vortex filaments, i.e. flux lines, (see Fig. 2) and their consolidation on the defects present there $[55,56]$. Such filaments may also remain in the superconductor at fields less than $H c 1$, and even at zero field if it is achieved by lowering from the area of larger fields, $H>H c 2$. This is associated with the arising induction ('Meissner') currents flowing around the superconductor surface. They seek not only to escape the penetration of external magnetic fields into the superconductor but also prevent the magnetic field vortex filaments (field lines) from releasing of its thickness, those ones that penetrated the sample at temperatures $T>T c$ when it was not superconducting yet. This also determines, in contrast to type 1 superconductors, the irreversible (hysteretic) magnetization curve for type 2 superconductors being in Abrikosov condition, predicted theoretically by K.P. Bin and J.D. Livingston, which is practically very diverse (Fig. 4, [38]). At temperatures close to the critical temperature of superconductivity $T c$, the hysteresis phenomenon almost disappears.

Along with the critical external magnetic field there is also the critical current which limits the superconductivity due to creation of the self-magnetic field of critical value with induction $B c=\mu_{0} H c$. Its energy 

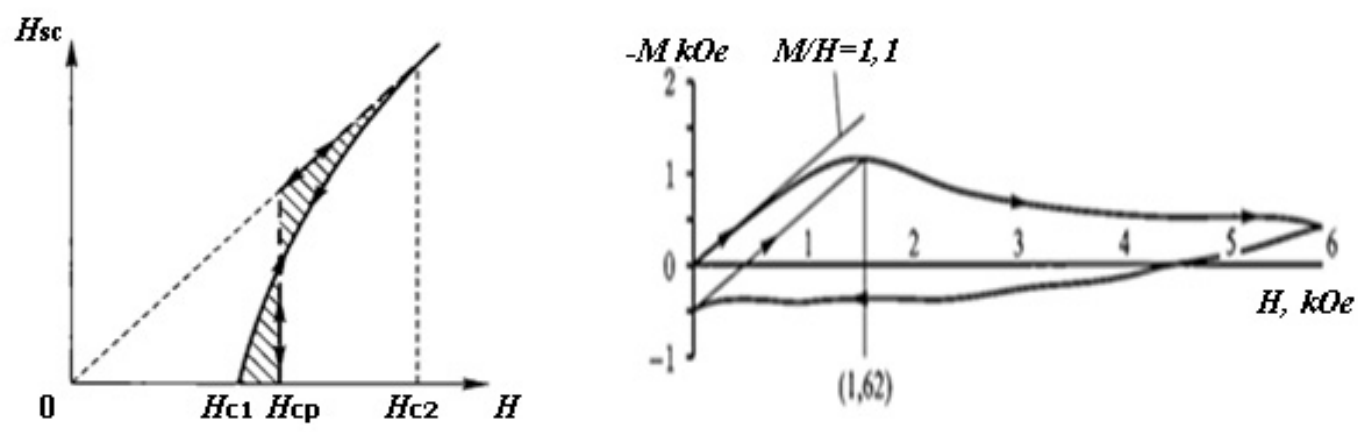

FIG. 4. External magnetic-field dependence of the magnetic $H$ intensity in ideal hard and soft superconductors with the same thermodynamic $H_{\text {ave }}$ - critical field (a), and the actual magnetization curve conforming to the sample (b). Herein, $M=p m V$ is for the magnetization vector; $p m$ is for the magnetic moment vector; $V$ is for the volume [38]

$W=m u_{0} H^{2} c / 2=B^{2} c / 2 \mu_{0}=G n(T)-G c(T)$ is thermodynamically related to the difference of free Gibbs energies of the normal $G n(T)$ and the superconducting $G c(T)$ states, regardless of whether the critical current only escapes the external magnetic field or it is a transport current that does not depend on the field. The excess of energy in magnetic line bonds to the centers of their spinning consolidation during the current flow, when the Lorentzian force influencing the vortices reaches a critical value, leading to an immediate change of their position (the magnetic flux jump) accompanied by a simultaneous temperature rise which may be sufficient to create the normal state of the superconductor [57].

The disturbance of this equilibrium but naturally metastable state of the superconductor vortex lattice with a trapped magnetic flux can probably occur during the flow of weak currents of subcritical value under the triggering action of external perturbations, including thermal and magnetic fluctuations of small intensities. However, unlike the previous case, the process of magnetic flux redistribution (so-called magnetic flux creep) in massive superconductors is long enough at a typical speed of thermal fluctuations. It manifests itself in a slow creep (in the perpendicular direction to the current density vector and the magnetic field induction vector) of pinned mutually repelling vortices to their general 'Abrikosov' state, with an equally slow magnetization relaxation to the equilibrium logarithmical value in time [58]. J. Giaever [59] carried out a direct observation of such movement of the niobium particles sawed on a lead foil. In all cases, the irreversible processes accompanied by heat release due to the energy dissipation of superconducting current, take place in the superconductor. Phase diagrams for superconductors of various kinds during tension $H$ change of an external magnetic field and temperature $T$ are schematically represented in Fig. 5 (based on [60,61]).

Experimental studies on the magnetization structure and dynamics for different kinds of superconductors with different composition stoichiometry, of different shape and size have been carried out in many scientific centers [62] by means of magnetic neutron diffraction and magnetic decoration (under the electron microscope by coating the superconductor surface with tiny magnetic iron or nickel particles). They have allowed 'visual' illustration of these processes, including the emergence of intermediate-mixed ('semi-Meissner') state and assessing their energy performance.

Another coherent effect of superconductivity should be noted. It lies in the specific tunnel junction of superconducting pairs from one superconductor to another through a very thin dielectric layer separating them, when a current passed is less than the critical value, as well as in the voltage drop in their contact with the simultaneous emission of electromagnetic waves when a current passed is greater than the critical value [63, 64]. The effect was predicted in 1962 by B. D. Josephson [65] based on the BCS theory. It was confirmed experimentally for the stationary case in 1963 by P. Anderson and J. Rollo [66], although the same phenomenon of resistance disappearance in the small contact between the two metals during transition to the superconducting state was observed in 1932 by W. Meissner and R. Holm [4]. According to modern concepts [67], the microscopic mechanism for Cooper pair tunneling is associated with the so-called 'Andrey's' reflection $[68,69]$ of quasiparticles localized in the potential well in the contact area. Thus, there is a change of signs of the effective mass and the charge carriers along with the transformation of an electron into a hole or vice versa and Josephson current value can achieve the value of a normal tunneling current through contact at the gap range stress in the superconductor energy spectrum [67]. 

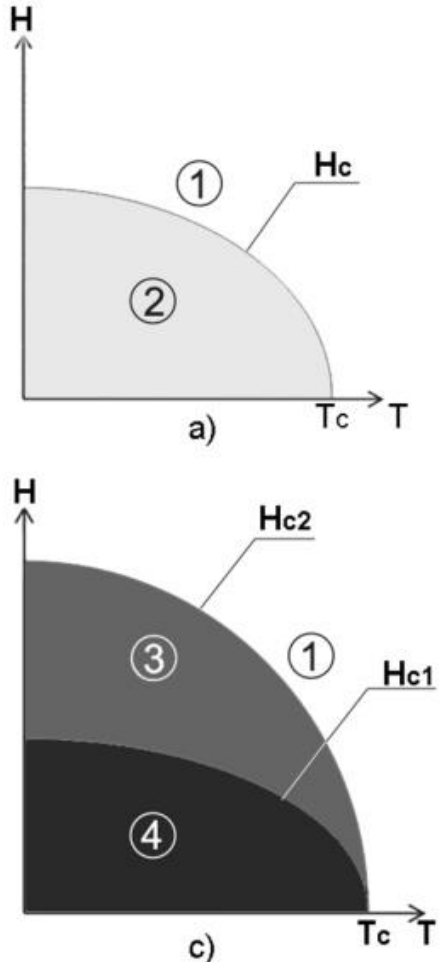

c)
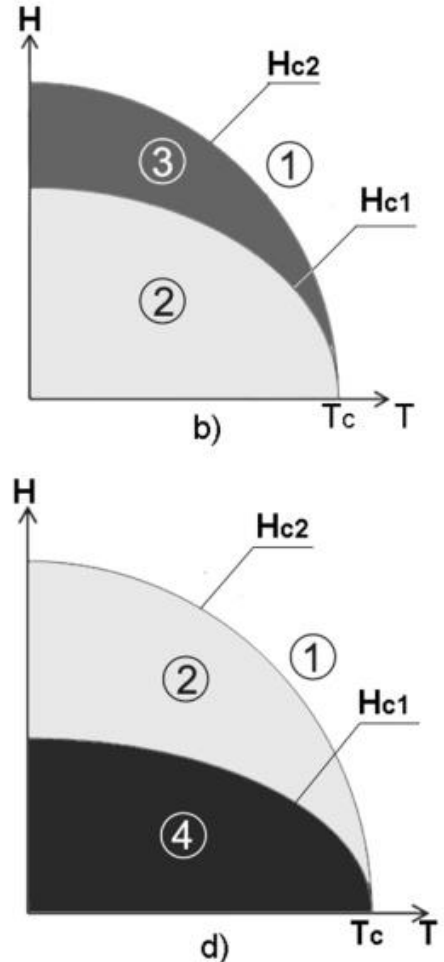

d)

FIG. 5. Schematic state phase diagrams for various superconductors (based on [60,61]): a) type 1 superconductors; b) type 2 superconductors; c) and d) - probable phase diagram for type 1.5 superconductors in case of different two-gap state parameters. Diagram section 1 shows the normal state, section 2 shows the Meissner state (the Meissner phase), section 3 shows mixed, or vortex state (the Schubnikov phase), section 4 shows the state characterized by a non-uniform vortex lattice

\section{Energy spectrum of superconducting materials, energy 'gap' and 'pseudo-gap' in HTSC}

Along with the above mentioned macroscopic effects in superconducting materials, there are also transformations at the microscopic level in the form of changes in the atom energy spectrum. The usual set of discrete electron energy levels in each atom that are allowed by quantum laws, fissionable to very large number of sublevels due to the huge number of atoms interacting with each other in a solid, almost merges into a continuous spectrum. Its feature for metals at temperatures above the critical lies in blocking the area of free 'conduction' electrons (those having more energy) and the area of bound 'valence' electrons (having lower energy values), whereas in case of semiconductors and insulators, these areas are separated by an energy gap (a band gap where there are no electrons). The coupling of electrons into Cooper pairs in metals at temperatures below the critical $T c$ leads to the emergence in their energy spectrum of the 'superconducting' energy gap, symmetric with respect to the Fermi level, of about $\Delta(T) \sim 10^{3} E_{F}$ width. It separates the equilibrium 'paired' electrons contained therein from other 'non-superconducting' particles which may be above or below the gap. This fact makes it different from the energy gaps in semiconductors or insulators where there are no electrons (Fig. 6).

Since the combined energy of a Cooper pair in the superconducting gap at the Fermi level is less than the total energy of two free electrons, in order to separate them back it is necessary to expend energy equal to the doubled gap energy $2 \Delta(T)=3.5 k T c$, the value of which is determined by the critical temperature $T c$. Therefore, the higher $T c$ is, the more energy is needed to break this pair. In this case, to break the rigidly connected system of all Cooper pairs an energy of $2 \Delta(T) \eta_{\text {coop }}$ will be required. But at temperatures above the critical value, the superconducting energy gap vanishes, $\Delta(T)=0$ and the Cooper pair density also becomes zero $\eta_{\text {coop }}=0$. The type of dependence of the superconducting energy gap width on temperature is shown in Fig. 7 for Al (Tc $\sim 1.18 \mathrm{~K})$ [38] and for $\mathrm{YbB}_{2}(T c \sim 115 \mathrm{~K})$ according to [72]. 


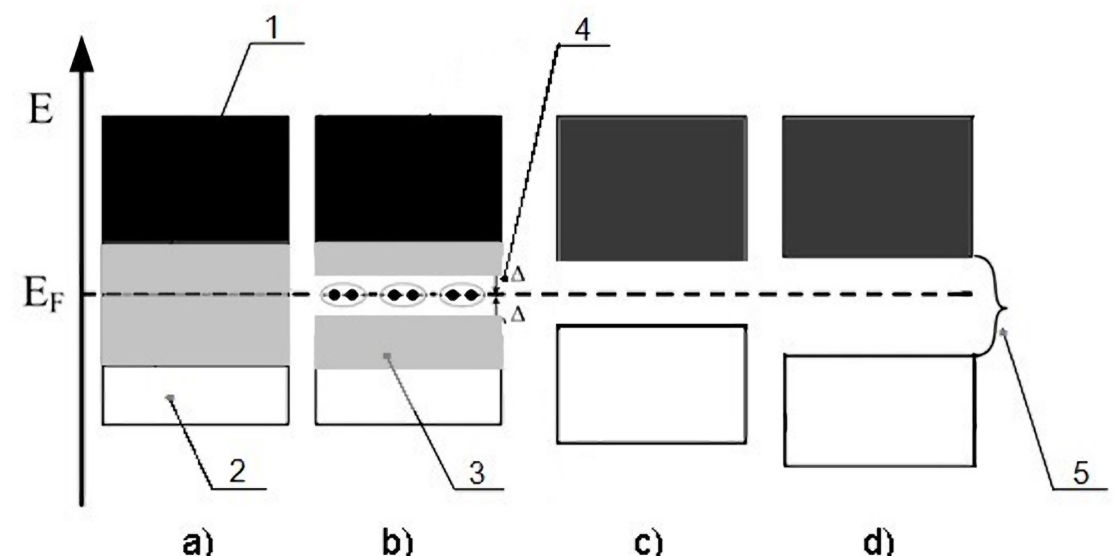

a)

b)

c)

d)

FIG. 6. Schematic image of the substance energy spectrum being in various states (based on Figures from [70,71]): a) - low-temperature superconductor at temperatures above $T c$ (normal state) and a normal metal; b) - low-temperature superconductor at temperatures below Tc (superconducting state); c) - semiconductor; d) - insulator; 1 - conduction band, 2 - valence band; 3 - mutual intersection (overlap) of both bands; 4 - symmetric superconducting ' $\Delta$ and $\Delta$ ' gap of the Cooper pairs (marked with ovals); 5 - band gap. $E_{F}$ is for the Fermi level energy

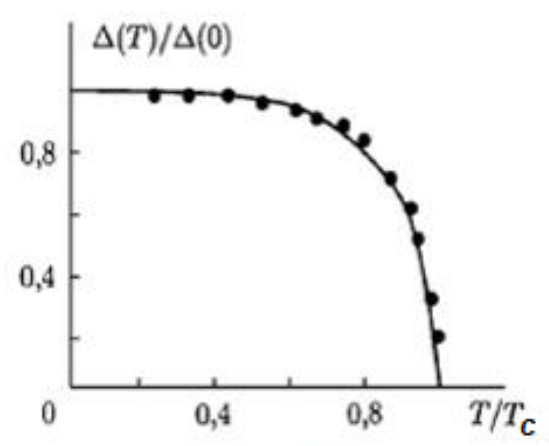

a)

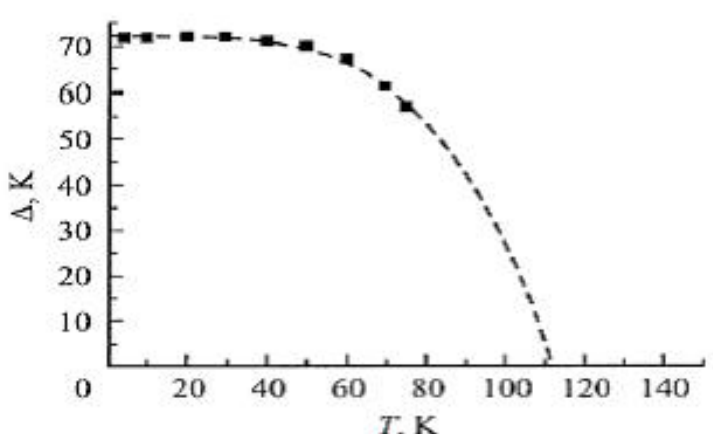

b)

FIG. 7. The energy gap width dependence on the temperature: a) - for Al in relative units according to [38]; b) - for $\mathrm{YbB}_{2}$ in absolute units according to [72]

The superconducting Cooper pairing has its own characteristics in samples placed in a magnetic field. This will happen in the case when the energy advantage of the transition from normal to superconducting state will exceed the energy cost caused by the displacement magnetic field. For type 1 conductors in fields which are larger than the critical value $H c 1$, the superconducting state is unprofitable and should be destroyed. However, as mentioned above, the possible distortion of the critical field due to the heterogeneous interaction with differently shaped conductor areas can lead to the simultaneous existence in the sample of sufficiently large areas of superconducting and normal phases separated by a substance in the 'intermediate' phase with the 'positive' surface separation energy obtained externally due to the supply of the magnetic field energy. At the same time, as noted above, when the field in the form of beams or individual vortex filaments penetrates into the type 2 superconductor, some of them may remain in such a superconductor in fields between $H c 1$ and $H c 2$, as well as in fields less than $H c 1$ and even in case of zero field if it is achieved by lowering from the area of larger fields, $H>H c 2$. Meanwhile, the conductor goes into the 'mixed' (metastable by nature) state along with preserving the superconducting properties and 'negative' surface separation energy into the largest possible number of normal and superconducting phases as a state that is energetically most favorable and does not require an external energy supply. 
However, the presence of impurity of magnetic atoms in the conductor significantly reduces the critical temperature of transition to superconducting state until it is completely liquidated, whereas non-magnetic impurities have only a small effect on the transition temperature [26]. We also determined that in the case of superconductors with magnetic impurities, the energy gap magnitude becomes zero somewhat earlier than the superconductivity disappears, since their energy spectrum has pairs with different binding energy including that arbitrarily close to zero. Such gapless superconductors [73] are characterized by several unusual properties, in particular, a linear heat capacity dependence on the temperature, as in case of normal metals. Superconductors with magnetic impurities possess other interesting properties, for example, the theoretical possibility for the existence of a phase that is simultaneously superconducting and ferromagnetic, which can also enhance the superconducting transition temperature [74].

It should be noted that in spite of the significant theoretical results, there have not been notable achievements in the practical search of materials with high transition temperature for the superconducting state for a long time. The 'warmest' superconductors from a large number of metals and hundreds of alloys identified, up to 1986, were considered to be the intermetallic films of niobium germanide $\mathrm{Nb}_{3} \mathrm{Ge}$ where $T c=23 \mathrm{~K}$. This value was the maximum one which had been managed to achieve for the critical transition temperature over 75-year research period, meanwhile the BCS theory did not predict the substances of a higher critical temperature. In the late 1960's to the early 1970's, great hopes were pinned on the organic complex synthesis with superconducting charge transfer, for example, complexes of TCNQ-TTF (tetracyanoquinodimethane-tetrathiafulvalene) [75-77]. However, despite the synthesis of a number of promising compounds, it was found that superconductivity in these complexes is unstable even at low current densities. Progress has become more significant only with introduction of the scientific paper by J.G. Bednorz and K. A. Muller who established in 1986, that ceramics of the $\mathrm{La}-\mathrm{Ba}-\mathrm{Cu}-\mathrm{O}$ compound based on oxides of copper, lanthanum and barium (compound $\mathrm{La}_{2-x} \mathrm{Ba}_{x} \mathrm{Cu}_{04}$ ) becomes superconducting at a temperature of $30 \mathrm{~K}$ [78]. Yu. D. Tretyakov specified this issue in his article 'Chemical superconductors before the Third Millennium' [79] '...The most striking fact was that it was not any particular organic or polymeric structure, on which theoretical physicists had pinned their hopes, that exhibited superconductivity, but oxide ceramics which is typically characterized as dielectric or superconducting. However, the most amazing fact for experts was that the oxide compounds exhibiting superconductivity had been synthesized long ago. In 1978, this was made by the IONKh RAN (Kurnakov Institute of General and Inorganic Chemistry of Russian Academy of Sciences) staff, namely V. Lazarev, B. Kahan and I. Chaplygin who published their results a year later in the "Journal of inorganic chemistry $(1979$, v. 24 . No. 6)"... They tried to investigate the synthesized samples but liquid helium was not available to them, likewise to the French researchers K.Michel and B.Pavo who synthesized similar and many other cuprates independently of their Russian colleagues. ...However, it goes without saying that Bednorz and Muller were not plagiarists, they discovered what they were purposefully and persistently looking for despite any predictions and theories'.

This discovery and the other one followed soon thereafter in 1987, concerning $93 \mathrm{~K}$ superconductivity in the $\mathrm{Y}-\mathrm{Ba}-\mathrm{Cu}-\mathrm{O}$ system $\left(\mathrm{YBa}_{2} \mathrm{Cu}_{3} \mathrm{O}_{7-x}\right.$ compound [80]) stimulated the search for copper-containing superconductors with a higher temperature all around the world. In 1988, the superconducting transition temperature of cuprate series with alkaline earth metals without rare earth elements in the $\mathrm{Bi}_{2} \mathrm{Sr}_{2} \mathrm{Ca}_{x-1} \mathrm{Cu}_{x} \mathrm{O}_{2 x+4}$ compound synthesized by the Maeda group, reached $108 \mathrm{~K}[81,82]$, and in case of cuprate materials in the $\mathrm{Tl}-\mathrm{Ba}-\mathrm{Ca}-\mathrm{Cu}-\mathrm{O}$ system $\left(\mathrm{Tl}_{2} \mathrm{Ba}_{2} \mathrm{Ca}_{2} \mathrm{Cu}_{3} \mathrm{O}_{10}\right.$ compound discovered by Sheng Z.Z. and Hermann A. M. in 1988) it was $125 \mathrm{~K}$ [83, 84]. The discovery of superconducting mercury cuprates in 1993 by S.N. Putilin and E. V. Antipov increased the transition temperature to $138 \mathrm{~K}\left(\mathrm{HgBa}_{2} \mathrm{Ca}_{2} \mathrm{Cu}_{3} \mathrm{O}_{8+x}\right.$ compound doped by $\left.\mathrm{Tl}\right)$, and under external pressure of 350 thousand atmospheres, the transition temperature increases to $164 \mathrm{~K}\left(-109{ }^{\circ} \mathrm{C}\right)[85,86]$. It is only $19 \mathrm{~K}$ less than the minimum temperature registered on earth. To date, there are more than 50 known original layered HTSC-cuprates [79].

Although the use of metal superconductors instead of ceramic ones solved the problem of increasing $T c$, it simultaneously created a problem of reduction of the critical $J c$ currents that destroy superconductivity. As noted by Tretyakov Yu. D. [79], the latter was the result of fundamental differences of metallic and ceramic superconductors. If the first ones are isotropic and have a coherence length of up to $20 \mathrm{~nm}$, the second ones are highly anisotropic and their coherence length does not exceed $2 \mathrm{~nm}$. As a result, in the polycrystalline condition, the crystallites' boundary in the ceramic superconductors is comparable with the coherence length and creates an effective barrier to the movement of magnetic vortices and increases the critical current, which is very important in technical applications, for example, to obtain strong magnetic fields. In particular, in the systems that generate, accumulate, and transport electric current, in magnetic cushion transport or in NMR-scanners, superconductivity must be maintained at currents of about $10^{5} \mathrm{~A} / \mathrm{cm}^{2}$ in a magnetic field of 2 to $10 \mathrm{~T}$, while the first samples of superconducting cuprates obtained using the traditional ceramic processes, had rather small critical current value 
of about $I c \sim 1 \mathrm{~A} / \mathrm{cm}^{2}$ [87]. Due to the high responsiveness of this parameter to the structural characteristics of superconducting ceramics, in order to prepare and treat it with heat, at the P. Makdgin's [79] suggestion they began to apply melt technologies used traditionally in the manufacture of metal but not ceramic materials. This became possible due to exceptional high fusibility of superconducting cuprates compared to the conventional oxide ceramics based on $\mathrm{MgO}, \mathrm{Al}_{2} \mathrm{O}_{3}$ or $\mathrm{ZrO}_{2}$. However, due to incongruent nature of melting (melting with decomposition where the solid phase is converted into a melt and solid phase of another compound), the melt product is not single-phased as a rule. However, the presence of inclusions of non-superconducting phases responsible for the emergence of additional pinning centers, i.e., braking of the magnetic vortices, contributes to a significant increase of superconductive stability in a magnetic field. In this case, the peak effect revealed in rare earth ceramic system $\mathrm{Ln}-\mathrm{Ba}-\mathrm{Cu}-\mathrm{O}(\mathrm{Ln}=\mathrm{La}, \mathrm{Nd}, \mathrm{Sm}, \mathrm{Gd}, \mathrm{Eu})$ [79], occurs at the same time. The nature of pinning associated with the peak effect, is fundamentally different from the pinning due to the introduction of hetero-phase non-superconducting inclusions. Based on the study of microstructural phase changes related the peak effect in the system, it is presumably associated with the formation of nanoscale areas of solid solutions at the original substance spinodal decomposition during formation and cooling of the melt enriched with barium and copper oxides [78]. The discovery of this effect gave rise to hopes for a radical improvement in technology and process parameters of superconducting cuprate materials which have recently been given considerable attention (see, for example, papers [88-91]).

Along with this, research was conducted on superconductivity of non-cuprate compounds, in particular, of $\mathrm{Mg}-\mathrm{B}, \mathrm{Ba}-\mathrm{Na}-\mathrm{Ge}, \mathrm{Na}-\mathrm{WO}_{3}$ systems [31,92-96], as well as iron compounds, ferronickel and iron selenides [97110]. To date, there is a wide class of such materials which give new opportunities to further increase the transition temperature of the superconductivity with the simultaneous increase in the critical current value. These data give reason to believe that in case of both copper and non-cuprate superconductors, layered materials including nanostructured ones are the most promising.

The anisotropy of physical properties along and across the layers, which determines the peculiarity of the superconducting Cooper pairing, has been shown to play a special role in increasing temperature of the superconducting transition for all layered compounds [111]. In particular, discovered in 2001, superconducting $\mathrm{MgB}_{2}$ compound of the alkaline earth metal $\mathrm{Mg}$ and wide bandgap semiconductor B with alternating magnesium and boron layers and a transition temperature of $T c \approx 40 \mathrm{~K}$, a record for borides, has two superconducting gaps were predicted theoretically and confirmed experimentally $[112,113]$. In quasi-two-dimensional Bor hole bands $(\sigma$-bands) the superconductivity transition forms a $\Delta E \sigma$ gap in the quasiparticle spectrum, which is a band of forbidden energies for single electrons and of holes with values of about $10-11 \mathrm{MeV}$ at the maximum $T c$. The superconducting gap $\Delta E \pi$, forbidden band of approximately $1.5-3 \mathrm{MeV}$ width, is also formed in three-dimensional magnesium bands ( $\pi$-bands). The interaction of these two 'varieties' of Cooper pairs in two-band MgB2 material with the double-gap superconductivity ensures a high $T c$. As noted above, the doping leads to a decrease in the critical transition temperature $T c$ while increasing the critical current [92]. High-temperature superconducting state was also experimentally observed on surfaces of alkali metals, in particular, localized spots of the surface superconducting phase were found on the superficially doped crystal of $\mathrm{Na}-\mathrm{WO}_{3}$ at a critical temperature of $91.5 \mathrm{~K}[94,95]$.

An alternating layered structure is also characteristic for a new class of high temperature superconductors discovered in 2008 by a H. Hosono's group [97,98], i.e. layered iron compounds and pnictides (group V elements in the periodic table: nitrogen, phosphorus, arsenic, antimony, bismuth, etc.), or chalcogenides (group VI, main subgroup elements: oxygen, sulfur, selenium, tellurium, polonium). Compounds $\mathrm{LnFeAsO}_{1-x} \mathrm{~F}_{x}$ ( $\mathrm{Ln}=\mathrm{La}-$ Gd), in particular, $\mathrm{La}\left[\mathrm{O}_{1-x} \mathrm{~F}_{x}\right] \mathrm{FeAs}$ where $T c=26 \mathrm{~K}$ [97,98], $\mathrm{Sm}\left[\mathrm{O}_{1-x} \mathrm{~F}_{x}\right] \mathrm{FeAs}$ where $T c=55 \mathrm{~K}$ [99], $\mathrm{Nd}\left[\mathrm{O}_{1-x} \mathrm{~F}_{x}\right] \mathrm{FeAs}$ [100], $\mathrm{Gd}_{1-x} \mathrm{Th}_{x} \mathrm{FeAsO}$ where $T c=56 \mathrm{~K}$ [101] were synthesized on this basis, demonstrating for the first time the superconductivity of materials containing magnetic components of $\mathrm{Fe}$ atoms in alternating layers, despite screening by a tetrahedron of As or Se atoms. Subsequently, superconducting Fe compounds were synthesized with Ce, As, F, As, K, Se, Te, Rb, K, Na and other elements [102-108].

The energy spectrum of each band in substances with such multi-band structure has its own gap, which leads to at least two superconducting bosonic condensates and multi-gap superconductivity similar to the case of magnesium diboride [109-112]. The presence at the Fermi level of two conduction electron types ( $\pi$ - and $\sigma$-electrons) of different dynamical (effective) mass which is manifested in the particle motion in the electric field of the crystal lattice of each structural area of such material, leads to different width of each of the superconducting energy gaps $\Delta \pi$ and $\Delta \sigma$, to different superconductivity types (type 1 for paired $\pi$-electrons and type 2 for $\sigma$-electrons), and to the divergence of other coherence characteristics. Now there are several collections of iron-based superconductors, including new compounds created in FIAN (P. N. Lebedev Physical Institute of the Russian Academy of Sciences) from barium, iron and 122-type arsenic. The materials of this group are being studied very actively now, their 
production is simpler than the synthesis of their analogues in other collections, for example those consisting of gadolinium, arsenic, iron, and oxygen, such as GdOFeAs doped with fluorine which replaces the oxygen [113].

In accordance with the early theoretical two-gap superconductor models proposed by V. A. Moskalenko and H. Suhl $[114,115]$ independently, without interband interaction, both gaps behave accordingly with BCS theory and become zero at their own critical temperatures $T c^{L}$ and $T c^{S}$. And by having this interaction as shown by experiments $[116,117]$, both gaps go to zero when a $T=T c$ is the same and when $T<T c$, one of the gaps is more than the BCS-value and the other is less (Fig. 8).

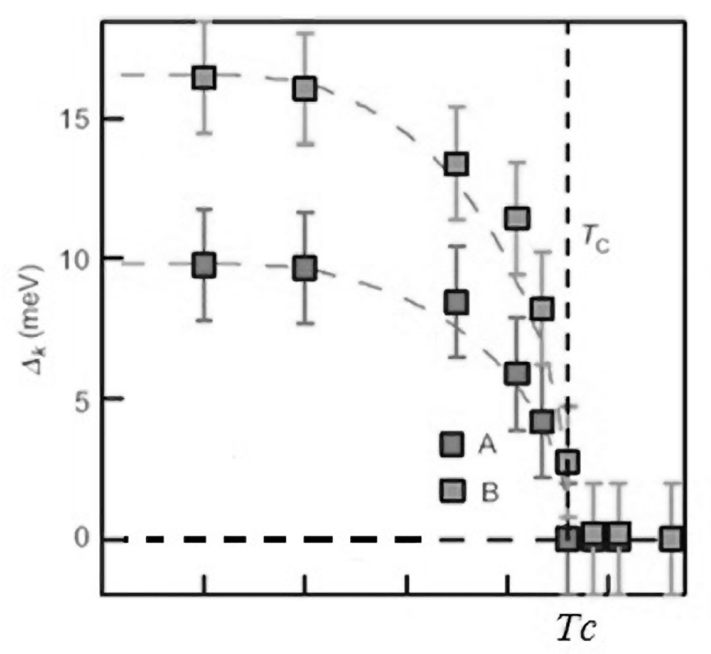

FIG. 8. The superconducting gap dependence on temperature in the two-gap HTSC $\mathrm{Bi}_{2} \mathrm{Sr}_{2} \mathrm{CaCu}_{2} \mathrm{O}_{8+x}$ [117]

During further development of the theory (I. I. Mazin, [118]) they proposed an alternative mechanism of Cooper pair formation, based on the magnetic resonance of dynamic magnetic susceptibility and then they suggested the interband interaction model for spin and even orbital fluctuations [120].

Type 1 and 2 phase transitions occurring in the two-gap superconductors with increasing temperature, change state with a non-uniform vortex lattice to a state with a regular (triangular) vortex lattice depending on the external magnetic conditions, then the condition of unevenly spaced vortices with a non-uniform vortex lattice can be observed again and, finally, they change to the Meissner state [121]. These phenomena, discussed above in connection with the superconductor transitions from one type to another when the magnetic field is changed, cause a very unusual kind of hysteretic magnetization curve (Fig. 9).

The specificity of 'iron' superconductors lies in the fact that in superconductivity, they exhibit another, antiferromagnetic, phase transition which transforms the magnetic moments of the substance's atoms (in this case neighboring Gd atoms) into the state of oppositely directed and equal in magnitude, which reduces the total material magnetization to zero. This magnetic condition is opposite in a way to a condition of the ferromagnetic materials where, if the temperature is below a certain critical value (the Curie point), magnetic moments of all atoms are directed in one way resulting in the ability to become magnetized in the absence of an external magnetic field. The substance becomes antiferromagnetic at the temperature being below a certain $T_{N}$ value (called the Néel point) and remains antiferromagnetic up to $T c$. It should be noted that the anti-ferromagnetism emerges in these and many other compounds at high temperatures as a phase that precedes superconductivity at a higher temperature, or in the 'ancestor' non-doped compounds. As a number of theorists believe, this means that the 'glue' that connects the electrons into superconducting pairs, is their interaction with magnetic fluctuations [122].

In 2009 - 2014 anoxic iso-structured layered selenides $\mathrm{A}_{x} \mathrm{Fe}_{2-Y} \mathrm{Se}_{2}(\mathrm{~A}=\mathrm{K}, \mathrm{Rb}, \mathrm{Cs}, \mathrm{Te}, \mathrm{Tl}$ etc. $)$ were obtained, among which there are superconducting compounds [104-108,123]. Superconducting properties are found in the $\beta$-phase of iron monoselenide $\mathrm{FeSe}_{1-\delta}$, with a significant non-stoichiometry of the chalcogen $\delta \sim 0.18$. From the phase diagrams of the system $\mathrm{Fe}-\mathrm{Se}-\mathrm{Te}$, it follows that tetragonal modification of the $\beta$-phase is stable at temperatures ranging from room temperature to $\sim 844{ }^{\circ} \mathrm{C}$, while that in the system $\mathrm{Fe}-\mathrm{Se}$ is stable to $\sim 457{ }^{\circ} \mathrm{C}$ (Fig. 10, [124]). To obtain SC-samples in the $\mathrm{Fe}-\mathrm{Se}$ and $\mathrm{Fe}-\mathrm{Se}-\mathrm{Te}(\mathrm{S})$ systems, both standard material science methods and less time consuming ways of obtaining poly- and monocrystals [125]. FeSe has a much simpler crystal structure, but it has many of the same magnetic and electronic properties (including superconductivity) as the iron-based pnictide superconductors (Fig. 11, according to [126]). 


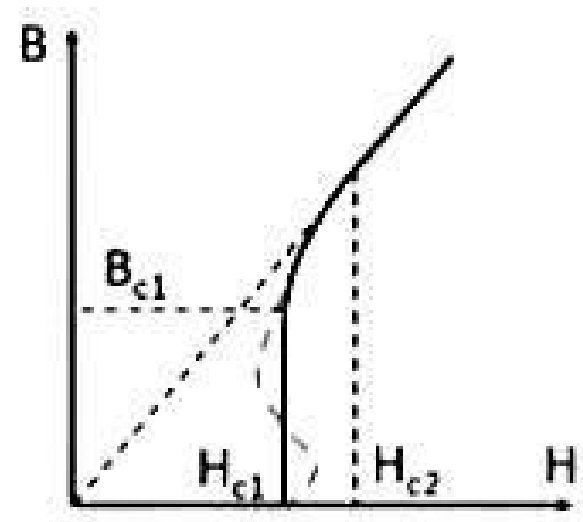

FIG. 9. The magnetic induction dependence on the magnetic field applied and the magnetization hysteresis for two-gap superconductors (schematically, according to numerical simulation of systems such as $\mathrm{MgB}_{2}$ and layered Fe-containing compounds [121])

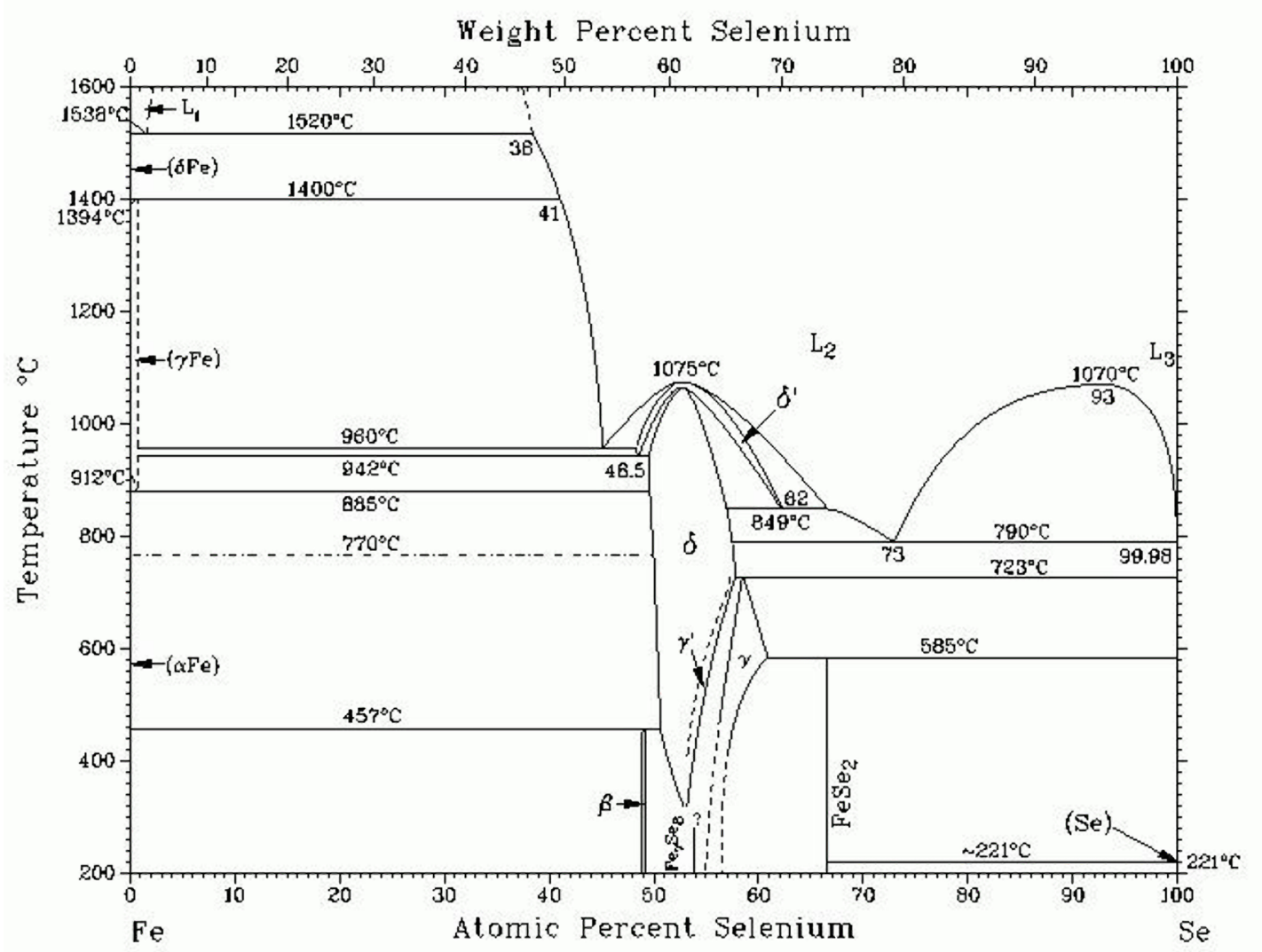

FIG. 10. The phase diagram of the system Fe-Se according to [124]. Superconducting properties are found in the $\beta$-phase of iron monoselenide $\mathrm{FeSe}_{1-\delta}$

The critical magnetic field which such compounds are able to withstand while remaining a superconductor, according to experts from FIAN (P. N. Lebedev Physical Institute of the Russian Academy of Sciences) is a great value about $130 \mathrm{~T}$ [38]. This parameter, in addition to the other two ( $T c$ and $J c$ ), is very important for technical applications. As for the new compounds, it almost matches the achieved value of $H c 2=100 \mathrm{~T}$ for the most studied 


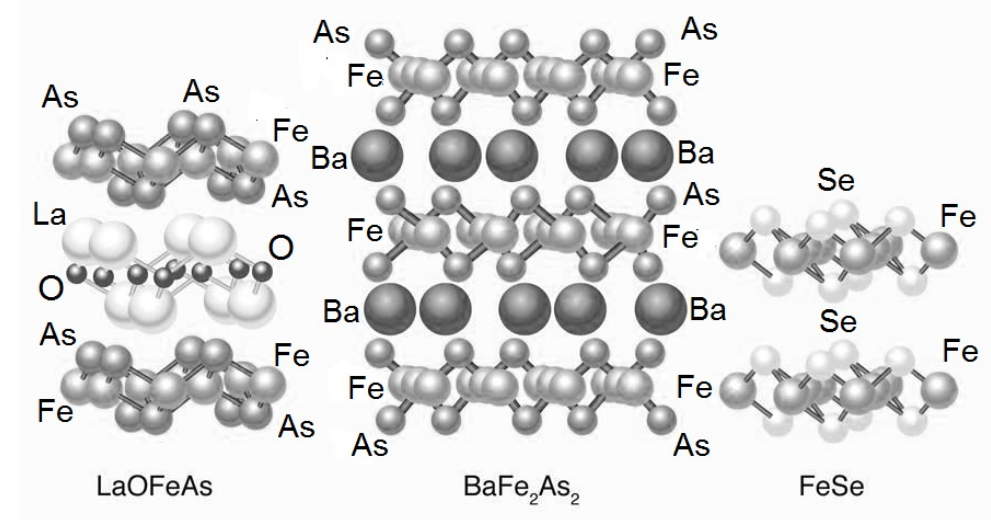

FIG. 11. Comparison of the crystal structures of the iron-based pnictide superconductors on the example of $\mathrm{LaOFeAs}$ (left) and $\mathrm{BaFe}_{2} \mathrm{As}_{2}$ (center) and FeSe (right), based on [126]

TABLE 1. Electrical and technical specifications of some commercially available superconductors (according to [38])

\begin{tabular}{|c|c|c|c|}
\hline Compounds & $T c, \mathrm{~K}$ & $J c, \mathrm{~A} / \mathrm{cm}^{2}$ at $4.2 \mathrm{~K}$ & $H c, T$ at $4.2 \mathrm{~K}$ \\
\hline $\mathrm{NbTi}$ & $9.5 \div 10.5$ & $(3 \div 8) 10^{4}$ & $12.5 \div 16.5$ \\
\hline $\mathrm{Nb} 3 \mathrm{Sn}$ & $18.1 \div 18.5$ & $(1 \div 8) 10^{5}$ & $12 \div 13$ \\
\hline $\mathrm{NbN}$ & $14.5 \div 17.8$ & $(2 \div 9) 10^{7}$ & $8 \div 13(25$ at $1.2 \mathrm{~K})$ \\
\hline
\end{tabular}

TABLE 2. Parameters of some HTSC materials (based on [128, 129])

\begin{tabular}{|c|c|c|}
\hline Compounds & $T c, \mathrm{~K}$ & Number of $\mathrm{CuO}_{2}$ layers \\
\hline $\mathrm{La}_{1.85} \mathrm{Sr}_{0.15} \mathrm{CuO}_{4}$ & 40 & 1 \\
\hline $\mathrm{YBa}_{2} \mathrm{Cu}_{3} \mathrm{O}_{7}$ & 92 & 2 \\
\hline $\mathrm{Bi}_{2} \mathrm{Sr}_{2} \mathrm{CaCu}_{2} \mathrm{O}_{8}$ & 95 & 2 \\
\hline $\mathrm{Bi}_{2} \mathrm{Sr}_{2} \mathrm{Ca}_{2} \mathrm{Cu}_{3} \mathrm{O}_{10}$ & 115 & 3 \\
\hline $\mathrm{Tl}_{2} \mathrm{Ba}_{2} \mathrm{Ca}_{2} \mathrm{Cu}_{3} \mathrm{O}_{10}$ & 122 & 3 \\
\hline $\mathrm{HgBa}_{2} \mathrm{Ca}_{2} \mathrm{Cu}_{3} \mathrm{O}_{8}$ & 134 & 3 \\
\hline
\end{tabular}

cuprate superconductor $\mathrm{Y}-\mathrm{Ba}-\mathrm{Cu}-\mathrm{O}$ having almost twofold critical temperature of $92 \mathrm{~K}\left(\mathrm{YBa}_{2} \mathrm{Cu}_{3} \mathrm{O}_{7}\right.$ compound) and $\mathrm{Bi}_{2}\left(\mathrm{Sr}_{2} \mathrm{Ca}_{2}\right) \mathrm{Cu}_{3} \mathrm{O}_{10}$ having $T c=110 \mathrm{~K}$ but considerably lower critical current. Meanwhile the maximum magnetic field obtained by the 'traditional' commercially available superconductors $\left(\mathrm{NbTi}, \mathrm{Nb}_{3} \mathrm{Sn}\right.$ and $\left.\mathrm{NbN}\right)$ is now fivefold smaller and only reaches 25 - $28 \mathrm{~T}$ (Table 1 [38]).

The theoretical assumptions concerning the definite role of increasing the number of layers for enhancing the critical $T c$ temperature, made on the basis of the effect of Josephson tunneling of Cooper pairs between the layers of HTSC materials [127], initially received an experimental confirmation in experiments with the homologous cuprate series (Table 2, based on [128, 129]).

Along with this, without using any behavior patterns for electrons in a normal or superconducting state, in his study, A.J. Leggett [129] derived a simple formula for estimating the critical temperature change depending on the $n$-number of $\mathrm{CuO}_{2}$ layers in HTSC families of the same structural type in comparison with a single-layer specimen from the same homologous series as the inequality $T c^{(n)}-T c^{(1)}=T o(1-1 / n)$, where $T o$ is an individual constant for each family. In particular, this formula results in $\left[T c^{(3)}-T c^{(2)}\right] /\left[T c^{(2)}-T c^{(1)}\right]=1 / 3$, which was consistent with the available experimental data $0.25-0.34$ for homologous series of cuprate HTSC having a number of layers $n=2$ and $n=3$ (see, for example, Table 2).

However, the subsequent experiments with a multi-layered metal oxide HTSC [130,131] by methods of scanning quantum-interference microscopy (SQUID) and nuclear magnetic resonance (NMR) showed that the 
Josephson's interlayer tunneling is not a basic mechanism for high-temperature superconductivity. The bellshaped curve $T c(n)$ with the cascading branch when increasing the layers number greater than $n>3$ and the redistribution of charge carriers from 'internal' to 'external' layers within one unit cell was also identified. The proposed theoretical model, that takes into account the last factor on the phenomenological level [132], describes the behavior of $T c(n)$ with a maximum at $n=3$ quite well (Fig. 12).

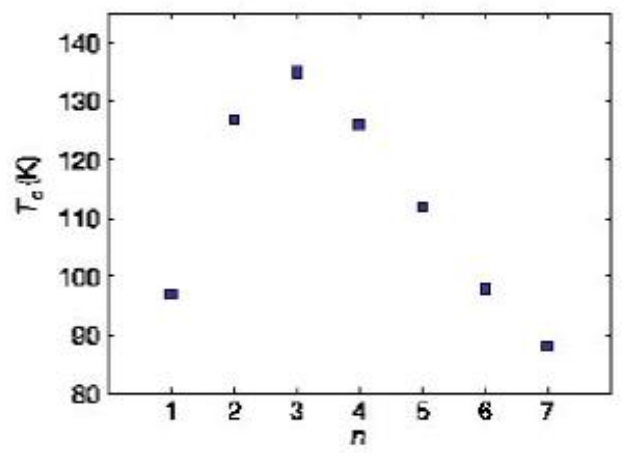

FIG. 12. The temperature dependence of the superconducting transitions on the number of $\mathrm{CuO}_{2}$ planes in the unit cell (schematically, according to [129] for homologous series of $\left.\mathrm{HgBa}_{2} \mathrm{Ca}_{n-1} \mathrm{Cu}_{n} \mathrm{O}_{2 n+2+\delta}\right)$

It should be noted that the nature of superconductivity in high-temperature materials produced is still not fully clear. They are not 'conventional' superconductors in terms of the Bardeen-Cooper-Schrieffer theory, for which the predicted critical temperature should not exceed the above limit of about $40 \mathrm{~K}\left(-233{ }^{\circ} \mathrm{C}\right)$, and the prospects in this area for achieving even a higher transition temperature are not clear. However, according to this theory, all that is required to achieve a high $T c$ is a favorable combination of high frequency phonons, strong electron-phonon bonding, and high density of states [133]. As recently shown by A.P. Drozdov, M.I. Eremets, I. A. Troyan, V. Ksenofontov and S. I. Shylin [134], these conditions can be theoretically fulfilled for metallic hydrogen and covalent hydrogen-dominated compounds. This idea was confirmed by computational studies predicting the transition temperature in the range of $50-235 \mathrm{~K}$ for many hydrides, in particular for hydrogen sulfide $\left(\mathrm{H}_{2} \mathrm{~S}\right)$, which is transformed to a metallic state at a pressure of $90 \mathrm{GPa}$, and the critical temperature for transition into the superconducting state depends on the temperature at which the sample becomes compressed. The results of experimental studies concerning the influence of these factors on the hydrogen sulfide's resistivity and the transition temperature obtained by the authors in 2015 are shown in Fig. 13.
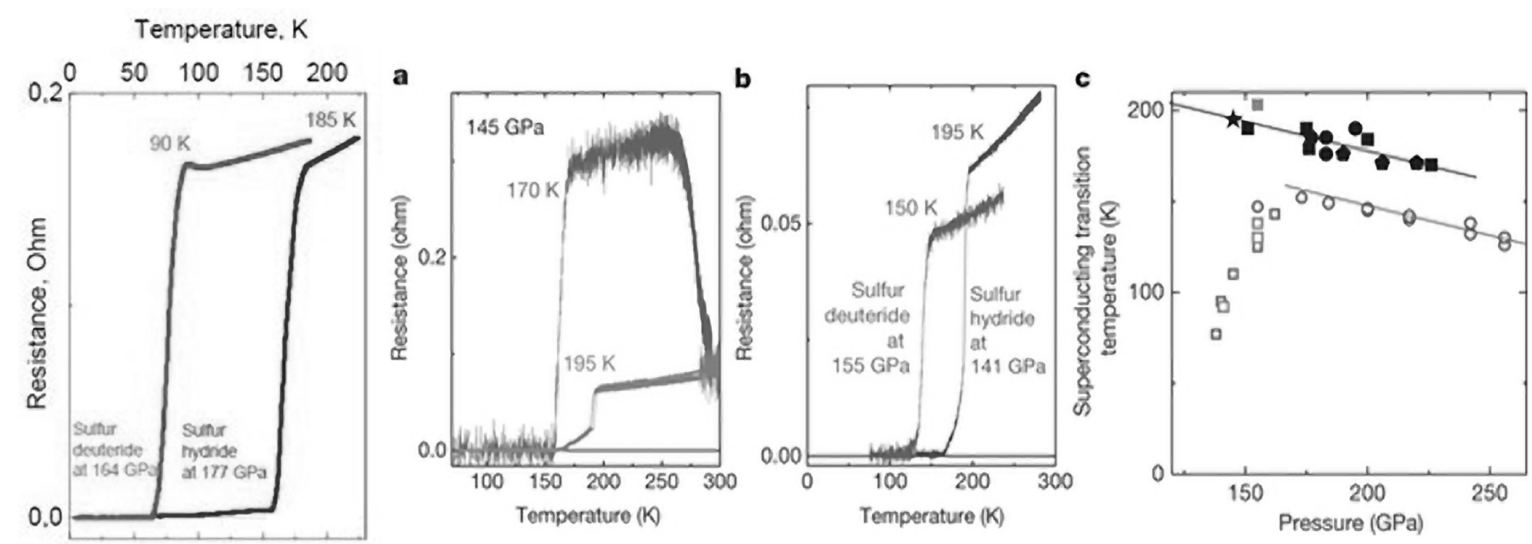

FIG. 13. The effect of sample pressure and temperature before compression on the electrical resistance and critical temperature of sulfur hydride and deuteride transition into the superconducting state (Figures taken from [134])

In their experiments, they recorded a new supercritical transition $T c$ temperature of about $203 \mathrm{~K}$ (or $-70{ }^{\circ} \mathrm{C}$ ) exceeding the previously noted record $T c$ level of $164 \mathrm{~K}\left(-109{ }^{\circ} \mathrm{C}\right)$ of 'unusual' (in the above sense) mercurycontaining superconducting cuprates for the compound $\mathrm{HgBa}_{2} \mathrm{Ca}_{2} \mathrm{Cu}_{3} \mathrm{O}_{8+x}$, which was achieved at an external 
pressure of $35 \mathrm{MP}$. The 'normal' hydrogen sulfide $\left(\mathrm{H}_{2} \mathrm{~S}\right)$ was compressed at high pressures of about $153 \mathrm{GPa}$ and a temperature of more than $220 \mathrm{~K}$, and, according to the authors, the superconducting transition was due to the formation of $\mathrm{H}_{3} \mathrm{~S}$ from $\mathrm{H}_{2} \mathrm{~S}$ by decomposition under pressure and strong isotopic shift of normal hydrogen to deuterium with the formation of $\mathrm{D}_{2} \mathrm{~S}$. The hydrogen sulfide transition into the superconducting state is confirmed by direct measurements through the presence of the Meissner effect using the method of nuclear resonant scattering of synchrotron radiation by the independent international research team [135]. There are predictions on the possibility to further increase $T c$ in the hydrogen sulfide based materials, for example, by replacing some sulfur atoms with phosphorus, platinum, potassium, selenium and tellurium; these predictions stimulate new directions of research on the hydrides superconductivity [135-139].

The above data on critical temperatures at which range of materials become superconductive, are compared to some characteristic temperatures in natural conditions in Fig. 14.

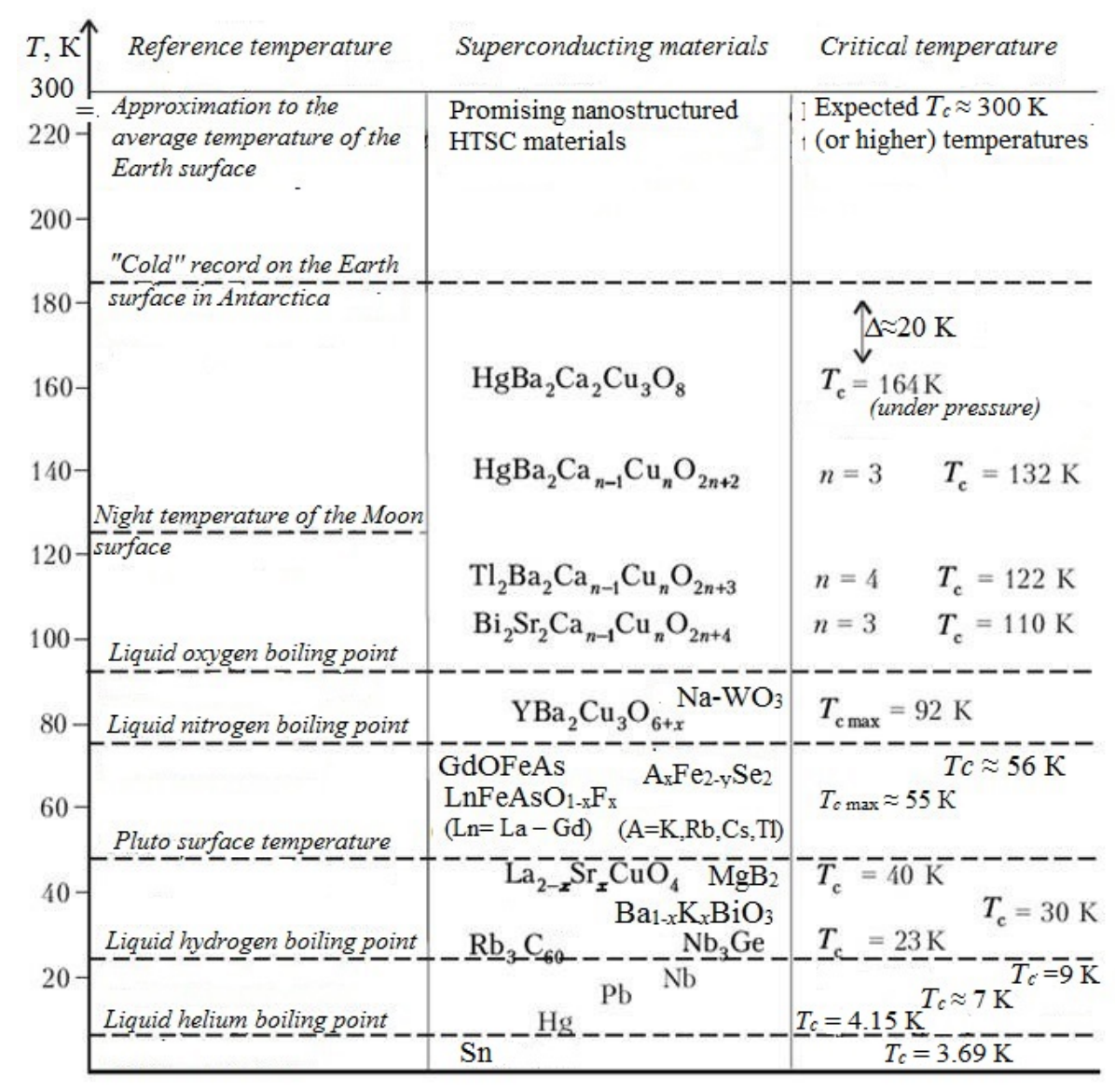

FIG. 14. Comparison of critical superconducting transition temperatures of some materials with representative temperatures existing in natural conditions (based on [29])

There are still no generally accepted results for the synthesis of more high-temperature superconductors, although there was some information about superconductivity in the materials based on diamond and other systems at a critical temperature close to room temperature [136-138]. At the same time, using different experimental techniques $[139,140]$ it was recorded that there is another energy gap emerging at a temperature above the critical, which was called as a 'phony' gap or a 'pseudo-gap' because of its inexplicable origin [30,137-143]. A number of researchers explain its appearance with the phase transition to a special 'pseudo-gap' state, and various hypotheses of the phenomena occurring during this process are considered in the proposed models. For example, it is argued that at temperatures above $T c$, Cooper pairs are not destroyed but only lose their coherence, i.e. lose the connection between them [144], or that a pseudo-gap at above the superconducting transition temperature appears due to the influence of clusters of superparamagnetic or antiferromagnetic ordering as a kind of 'magnetic glue' which occurs when a high-temperature superconductor is not doped or poorly doped with charge carriers [145]. It is also thought that such a mechanism lies in the formation of spin- or charge-density standing waves involving magnetically 
ordered structures of indirect exchange via the conduction electrons in their organization, leading to the formation of virtual quasi-localized states, but other ideas exist as well [143-148].

It is worth mentioning the opposing view of the same large number of researchers that see no relation between transition into the pseudo-gap state and any phase transition and explain it by suppression of the electronic states density of one-particle excitations near the Fermi level. This is indicated, in particular, by typical dependence of the superconducting gap characteristics on the external magnetic field, whereas the pseudo-gap practically does not depend on it $[149,150]$. This is evidenced by, for example, the data in the papers [117] and [151] which together show that at $T=T^{*}>T c$, a large spatially inhomogeneous gap (pseudo-gap), irrelevant to superconductivity, is formed in HTSC, while at $T=T c$, the superconducting gap is formed, which is uniform and much smaller in size.

These contradictions show that the authentic origin of the pseudo-gap is still unclear.

\section{Energy 'pseudo-gap' as an independent phase transition in high-temperature superconductors}

It is possible to distinguish the relationship of the energy pseudo-gap occurrence accompanied by formation of local incoherent Cooper pairs from other, competing with this, conditions by availability (in case of superconductive pseudo-gap) or absence (in another case) electron-hole symmetry of its energy spectrum relative to the Fermi level. However, it is clear that regardless of the specific mechanism, the emergence of the energy pseudo-gap can be considered as an independent phase transition in the substance prior to the transition to superconductivity in HTSC. Experimental evidence for this fact was presented by a large group of specialists from several research organizations in Japan, USA and Thailand [140]. Using a scanning tunneling microscope, they found that a significant role for the occurrence of this state is played by a critical level of doping of the superconductor $\mathrm{Ca}_{2} \mathrm{CuO}_{2} \mathrm{Cl}_{2}$ with sodium atoms, beyond which the material becomes superconducting at a rather high temperature. It was also revealed that when doping the original superconductor with sodium at very low levels, the atoms were observed to form in nanometer clusters which always appear during the pseudo-gap formation [152]. At the same time as the sodium atom concentration was increasing, their individual clusters began to merge gradually and when they were completely coupled, the material demonstrated the properties of superconductor.

The spontaneous appearance of thermodynamically non-equilibrium Cooper pairs (superconducting fluctuations) at temperatures above $T c$ results in, compared to conventional one-electron mechanism, the additional charge transfer known as 'Aslamazov-Larkin contribution' [153,154], as well as excess conductivity, or paraconduction [155]. Herewith, its conductivity and heat capacity, sound absorption factor, thermal EMF, Hall coefficient, anomalies of tunneling transition current-voltage characteristic, diamagnetic susceptibility and other phenomena can be significantly increased even in the normal phase of the superconductor material in the immediate vicinity above Tc. However, when the superconducting transition temperature is reached, the expected superconducting fluctuations, a consequence whereof is the effect of superconductivity, are not only suppressed but may be missing at all.

The common feature of all cuprate HTSC families with different types of doping is a bell-shaped critical $T c$ temperature dependence on the $x$-concentration of charge carriers (with a maximum at $x_{o p t} \sim 0.2$ for $p$-doping and at $x_{o p t} \sim 0.15$ for $n$-doping), as presented on the generalized phase diagram (Fig. 15) using papers [111, 156].

Considered in [157], the original explanation of this dependency within the theory of Josephson tunneling of Cooper pairs between $\mathrm{CuO}_{2}$ layers which, as already noted, also predicted an increase in $T c$ with increasing number of $\mathrm{CuO}_{2}$ layers in the unit cell [127], is not consistent with the above bell-shaped $T c$ dependence on the number of layers with a maximum at $n=3$ (see Fig. 12). The latter assumption about the relation of the possible mechanism of both effects with the 'Andreev' reflection is indirectly confirmed by the aforementioned NMR data in cuprate HTSC [131] which showed that in case of a large number $(n>3)$ of $\mathrm{CuO}_{2}$ layers, charge carriers are distributed non-uniformly, their concentration within a single unit cell in the 'internal' layers is smaller than in external ones. The results of the numerical modeling of these phenomena [158] based on phenomenological representation of the free energy of the system as a function of two interacting order parameters, superconducting and non-superconducting, indicate their possible connection with a certain ordering (probably, that of charge density waves) that competes with superconductivity. According to this widely discussed hypothesis in the literature (see, e.g., reviews $[143,144,157])$, the latter also determines the appearance of the pseudo-gap. In the above phase diagram (Fig. 15) it is located in the area of the current carrier concentration which is less than optimal and corresponds to the maximum $T c$ temperature, and reveals itself with a number of anomalies in their electronic properties in both normal and superconducting state. Due to the reduced carrier concentration in 'internal' layers of a multilayer unit cell, the pseudo-gap size is large in these layers, and the actual superconducting order is suppressed, which leads to decrease of $T c$ when $n>3$. This conclusion from modeling results confirms the competing contribution of the phenomena considered to the formation of superconducting correlations; however, it does not clarify the underlying mechanism of high-temperature superconductivity, by which, in particular, only 

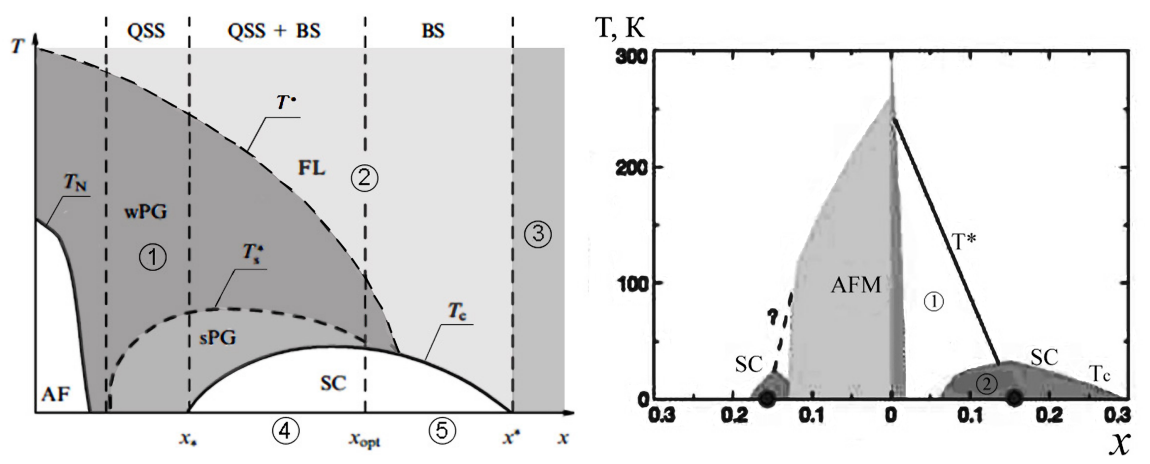

FIG. 15. Typical phase diagrams for cuprates of different hole and electron doping $x$-levels (according to $[111,156]$ ): a) - only hole doped ( $p$-type); b) - both $n$-doped (on the left, for the (La,Pr,Nd) ${ }_{2-x} \mathrm{Ce}_{x} \mathrm{CuO}_{4-y}$ compound) and $p$-doped (on the right, for the $\mathrm{La}_{2-x} \mathrm{Sr}_{x} \mathrm{CuO}_{4-y}$ compound). The figures indicate the following: 1 - a zone of incomplete doping, 2 - a zone of optimal doping, 3 - a zone of excessive doping, 4 and 5 - zones of poor and heavy doping, respectively. The letters indicate the following: FL - the normal Fermi-liquid area (normal phase); $\mathrm{SC}$ - the superconductivity area (the superconductivity phase); PG - the pseudo-gap phase area; sPG and wPG - strong and weak pseudo-gap areas, respectively; BS and QSS - areas of bound and quasi-stationary states of Cooper pairs, respectively, QSS+BS - area of their coexistence; AF and AFM - anti-ferromagnetism areas (antiferromagnetic phase); QCP - quantum critical point. Specific temperatures: $T c$ - the superconducting transition temperature; $T^{*}-$ the pseudo-gap transition temperature; $T s^{*}$ - the temperature of strong and weak pseudo-gaps crossover; $T_{N}-$ the antiferromagnetic transition temperature (the Néel temperature), $x$ - the doping level

a single $\mathrm{CuO}_{2}$ layer is superconducting. The question also remains open in terms of how to achieve a relatively uniform distribution of carriers in the layers so that the pseudo-gap deterring the critical temperature growth in superconducting macro-structures would not appear because of their unbalance.

\section{Peculiar properties of nanostructure superconductivity}

The possibility of superconductivity in metallic and non-metallic systems, and even in organic compounds at high temperatures was substantiated by V. L. Ginzburg [22,28] and W. A. Little [75-77]. They believed that the creation of room-temperature superconductors (RTSC) is one of the most important problems in the field of nanotechnology.

Fundamentally, this technology can be implemented by depositing atomic layers, for example, by atomic layer deposition (ALD), first proposed by V. B. Aleskovski in the mid-twentieth century $[159,160]$ and usually called as the method of molecular layering (ML) in the Russian scientific literature [161]. The specificity of this method lies in the possibility of forming a molecular or atomic monolayer on complex-shape substrates without forming threedimensional nuclei by cyclic alternate supply of gaseous (MOCVD - Metal Organic Chemical Vapor Deposition) or liquid (with subsequent pyrolysis - MOD - Metal Organic Deposition) precursor reagents of the buffer layer and the base material to the substrate in a way that the chemical reactions leading to the film growth occur only in chemisorbing layers, that is, with the exception of reactions in the gas phase. Meanwhile a given increase in the thickness of the formed film is easily and accurately controlled by the number of deposition cycles using the ability for self-organization (self-restraint) of surface reactions culminating automatically with the exhaustion of all reactive sites on the surface.

Another technological approach to produce the HTS conductors is the layer deposition in vacuum using the laser method (PLD - Pulsed Laser Deposition), electron or ion beam (Electron beam Deposition or Sputtering) and magnetron sputtering. Here, a high-temperature superconducting layer is deposited by ablation. When this technology is used, superconducting compound molecules or clusters, instead of individual atoms, should be transported from the target to the substrate and deposited on it $[162,163]$.

The mixed technology can be applied, for example, the buffer layers are applied by sputtering, and the HTSC layer is applied by chemical means.

In general, this enables the construction of both $2 D$-layered and $3 D$-dimensional superconducting nanostructures which are unique in terms of reproducibility and high conformity of the formed layers, thickness and density 
uniformity, number of defects, stoichiometric composition. A large variety of conductors and dielectrics can be used to generate the required electron-phonon spectra and obtain the superconductivity effect at a high temperature.

However, the existence of the superconductivity at high critical temperature in amorphous metal nanofilms [164] and in a number of nano-carbon substances synthesized based on insertion of the potassium atoms into fullerenes and nanotubes and the doping of a fullerene crystal with tribromomethane molecules [165, 166] shows that to obtain the superconductivity, the availability of an ordered structure similar to the layered cuprates or ferrous compounds is not required [167]. High-temperature superconductivity is typical of many other nanostructures. For example, it is typical of ultrathin $\mathrm{NbC}$ films and films from the aforementioned magnesium diboride, $\mathrm{MgB}_{2}$ [168], ultra-narrow silicon (Si) quantum $p$-wells bounded by $\delta$-barriers and heavily doped with boron [169], copper-containing fullerene quantum-well samples of endohedral nanostructures with the included element atom location inside the carbonic structure [170] and also of other nanostructures limiting the charge carrier movement in one, two, or three dimensions.

Discrete quantization levels of the charge carrier energy resulting from these limitations give rise to a great diversity of the superconductivity characteristics in the nanostructures compared to bulk samples of the same materials and open up opportunities for creating new materials as well. Thus, research on dependence of a critical value of the parallel $H c_{\|}$magnetic field for thin nanofilms superconductors $\mathrm{NbC}$ and $\mathrm{MgB}_{2}$ on their thickness and the mean free path of the electrons showed that at low temperatures the critical $H c_{\|}$field can significantly exceed the critical $\mathrm{Hc}_{2}$ field [168]. In case of $\mathrm{NbC}$ films, near the $T c$ critical temperature, the parallel critical $H c_{\|}$field varied depending on the film $d$ thickness in proportion of $\sim(1 / d)^{3 / 2}$, which is also the case for pure superconductors [171] and the observed temperature dependence of $H c_{\|}(T) \sim(1-T / T c)^{1 / 2}$ satisfy the superconductor vortex-free condition in the whole temperature range below $T c$. In a perpendicular magnetic $H c^{\perp}$ field, in case of both type 1 and 2 thin and ultra-thin superconductor plates being of thickness that is much less than the London penetration depth, the Meissner and the transport currents are almost constant by thickness. This leads to the transformation of the Abrikosov vortex into the so-called Pearl vortex [172] where the motion's speed can reach $103 \mathrm{~cm} / \mathrm{s}$ [173], as well as to the effect of local variations in the magnetic field direction (the 'vapor trail' in a magnetic field and even the 'contrail precursor' accompanying this vortex [174-176]). This effect reveals under the action of Lorentz force while passing both direct and alternating transport currents which make the vortices move uniformly or oscillate, and therefore, either keep fixed the shape of distribution of the vortex magnetic field in time or change it during the oscillation period while maintaining the general character of distribution (Fig. 16). Since the inversion of the longitudinal magnetic field component makes the vortices pull towards each other [177,178], the moving Pearl vortices in thin films of superconductors are arranged in chains which can be observed in magneto-optical experiments.

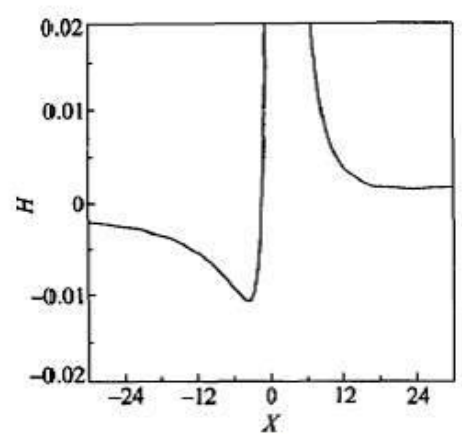

a)

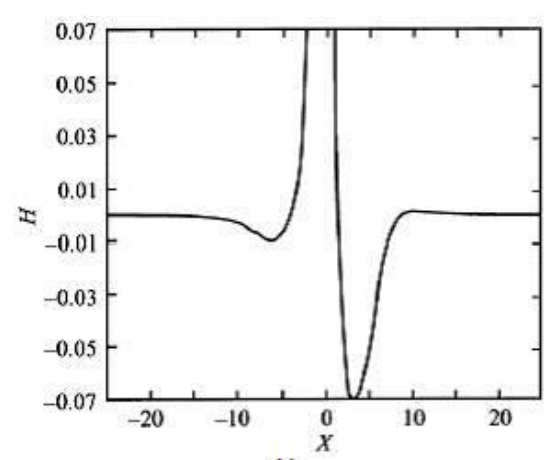

b)

FIG. 16. The distribution of stationary (a) and flash (b) magnetic field $H=2 \pi \lambda^{2} H c \perp / \Phi_{0}$ with respect to $X=x / \lambda$ in the direction of motion, respectively, for a uniformly moving Pearl vortex $[174,175]$; the minima correspond to the 'inversion precursor' in front of and to 'vapor trail' behind the vortex center. Herein, $x$ is for the distance from the vortex center, $\lambda-$ the London penetration depth, $\Phi_{0}$ - the magnetic flux quantum

Similar effects are also seen in the above-mentioned strongly anisotropic layered high-temperature superconductors, where the vortex structure is presented by vortices of two types, the properties of which differ markedly. The internal magnetic flux perpendicular to the superconducting layers is formed of point vortices merging into chains perpendicular to the layers, similar to Pearl vortices in thin films. Parallel to the layers, the magnetic flux is 
formed from vortices which are similar to vortices in tunnel transitions being parallel to the transition plates. The interaction between these vortices and the formation of composite vortices, which contain vortex segments of both types, leads to a complex dynamics of magnetic flux in strongly anisotropic layered superconductors [179].

Great opportunities to produce different types of self-ordering nanostructures such as quantum wires and crystallography-oriented quantum dot systems are provided by means of a method of molecular beam epitaxy [180], as well as ion implantation and non-equilibrium impurity diffusion methods [181]. As the method of atomic layer deposition (ALD) [182], they are widely applied in modern technology of semiconductors and nanoelectronics in the synthesis of other functional materials in planar technology [183-185]. In particular, created on this basis, the silicon sandwich nanostructures on the $\operatorname{Si}(100) n$-type surface which are ultra-narrow silicon quantum $p$-wells with high current carrier mobility, bounded by heavily boron-dope $\delta$-barriers, show high-temperature superconductivity with $T c=145 \mathrm{~K}$ and the superconducting gap value of $0.044 \mathrm{eV}$ [186]. As the authors suggest, superconductivity arises as a result of the transfer of hole ( $p$-type) bi-polarons of small radius through the dipole boron centers with negative correlation energy at the 'silicon quantum well $-\delta$-barrier' boundary. To create them, the fractal self-organization of the initial micro-defect distribution on the surface of oxidized single-crystal silicon [187] is applied; this process recurs during the quantum wells self-organization and, apparently, is a 'Sierpinski wipes'-type fractal modification [188] with an incorporated longitudinal silicon self-ordered quantum well (Fig. 17).

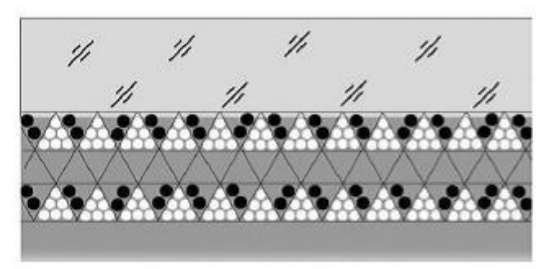

a)

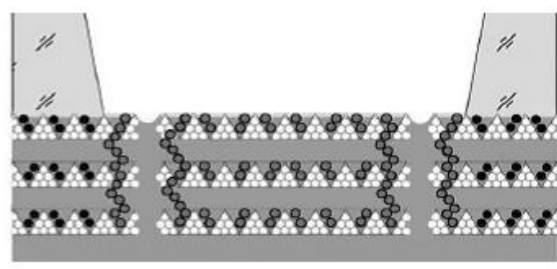

b)

FIG. 17. The diagram of self-ordered silicon wells produced on Si (100) surface under conditions of injection of the proper silicon interstitial atoms (white circles) and vacancies (black circles) during pre-oxidation (a) and subsequent micro-defect passivation under short-time boron diffusion by the vacancy mechanism (dark areas) (b) according to [187]

The superconductivity in $3 D$-fragments of carbon deposits with a high content of multivariable spatial nanotubular structures were discovered by V.I. Tsebro, O.E. Omel'yanovskii, and A.P. Moravskii from the Physical Institute of RAN (FIAN) (P. N. Lebedev Physical Institute of the Russian Academy of Sciences). They discovered almost undamped currents and magnetic flux capture at liquid helium temperatures, just as it occurs in a multivariable superconducting structure, moreover, the captured magnetic flux was damped very slowly even at room temperature [189].

Predicted theoretically, the phenomenon of oscillations in the critical temperature of the superconducting transition in the form of reentrant superconductivity in layered 'superconductor - ferrimagnet' nano-hetero-structures based on niobium and copper-nickel alloy is experimentally confirmed [190]. It may be considered as a direct proof that the inhomogeneous Larkin-Ovchinnikov-Fulde-Ferrell superconductivity (the above mentioned FFLO phase) occurs in the context of anomalous manifestation of the mechanism of 'paramagnetic' superconductivity destruction. In these experiments, samples of atomically-smooth thin film structures, produced using the known method of vacuum magnetron sputtering, were used.

The anomalous temperature-dependent diamagnetism existing within the range of room temperature to $750 \mathrm{~K}$ is discovered in aerosol-generated nickel oxide nanoparticles which were studied at the Institute of Structural Macrokinetics and Materials Science of the Russian Academy of Sciences during the crucibleless synthesis in the levitation-jet generator [191-193]. This effect is associated with the emergence of superconductivity on the surface of nanoparticles of averagely 10 to $30 \mathrm{~nm}$ in size and with different content of metallic nickel clusters. It was found that the thickness of the superconducting surface layer is independent of the particles size and is about $6 \mathrm{~nm}$ (approximately 30 atomic layers); however, in case of smaller particles, the anomalous diamagnetism disappears completely at room temperature. Evaluation of the second critical field for the superconductivity vanishing indicates the expected value of at least $15 \mathrm{~T}$ at $300 \mathrm{~K}$. Further consolidation of powder of these particles with the abovementioned magnetic behavior peculiarities was also studied applying the method of laser sintering under conditions ensuring the preservation of the superconductivity in the individual nanoparticles [194]. 
A promising way to create such layers with nanometer and sub-nanometer thicknesses and precisely-controlled chemical composition on the nanoparticle surface is the synthesis of composite 'core-shell'-structured nanoparticles. Both the above mentioned ML-ALD method of chemical layer deposition [195] and the method based on selforganization of nanoparticles of variable composition can be used for this purpose, for example, during the formation thereof in hydrothermal conditions due to displacement of the excessive components in composition from the resulting solid 'core' solution to a 'shell' that surrounds it [196-198].

The following progress of such technologies to achieve compact nanostructured superconducting materials is interconnected with both the development of new methods for the synthesis of 'core-shell' nanoparticles and new sintering methods in parallel with the study of individual nanoparticle interactions in the consolidated system. To solve the said problems and the problem of formation of nanomaterial structure and properties in general, the non-autonomous phases and corresponding non-autonomous substance condition play a particularly important role [199]. They are formed at the joints between macro-volumes of a substance as an intermediate transition surface or internal structures where a composition, a structure and properties are determined by space or mass limits, and which cannot exist as a separate thermodynamic phase. Apparently, the definition of a non-autonomous substance condition was given for the first time by Defaye and Prigozhin in their papers [200,201] in order to thermodynamically describe a substance which is localized in the boundary areas of volume phases and differs from them by its unique state equation (phase equation, according to J. W. Gibbs's terminology). The concept of 'nonautonomous phase' was used most successfully to describe the solid-phase processes (see for example [202-205]), although this approach also shows promise in the description of processes in the area of solid, liquid and gaseous phase contact [206-208]. Herewith, nanometer-scale environments with spatial limitations in one or several primary directions, where the walls neither exchange substance nor interact chemically with the substance localized in their limited space, can be considered as nano-reactors. The dimensional change of reaction zone affects the chemical or phase transformations occurring there (a known effect of reactor process scaling [209,210]. It is characterized by highly quick mass transfers in nano-reactors compared to their macro-sized counterparts. This, combined with the limited proportion of a substance contained there, provides a set of fundamentally new properties, in particular, the ability to save individual characteristics of nanoscale objects in the consolidated system with a great proportion of substance in the non-autonomous phases.

To study the emerging high-temperature superconductivity in ensembles of interacting nanoparticles [206], nanoscale approaches developed in earlier models of V. L. Ginzburg and W. A. Little [22,75-77] are involved, which are discussed in detail by L. V. Keldysh [211]. In particular, they proved the existence of the effect of superconductivity amplification during formation of various current contacts between nanoparticles of metals and alloys (tin, indium, lead, vanadium, zinc, lanthanum and their oxides) [208] where the delocalized electron spectra form energy shells similar to the atomic or nuclear energy shells [212,214-216].

The study of the superconductivity phenomenon at the level of interphase layers also indicates the possibility to create tunneling chains of metal nanoclusters with a total charge transfer [217]. This creates the chance to 'vastly' intensify the superconducting pairing and significantly increase the critical superconducting transition temperature up to the room temperature value [218]. This is caused by such a distinctive feature of nanoparticles as a discrete structure of their electron spectra due to the relatively small number of atoms compared to the massive volumes of regular solids. As a result, the superconducting energy gap value may exceed the difference between the energy levels that arise due to the finite particle size (a finite number of atoms in it) or come up with it [219]. In addition, as the energy levels are not equidistant, they can be highly degenerate and fairly close to each other in the vicinity of the Fermi level, which leads to the density peak of states at the Fermi level. According to estimates [220] detailed in [218], such a 'dimensional' quantization leads to the effect of $T c$ increase by value of $\delta T c / T c \approx(2-3)$, which was discovered in thin films and granular materials [220-224]. A similar situation in the clusters considerably stimulates the electron pairing; moreover, one can expect a tremendous intensify thereof compared to volume pairing, which emphasizes the importance of the nanoparticle shell structure for pairing as specified by W. Knight and several other researchers [225]. Most assuredly, this type of pairing also affects the optical, magnetic, thermodynamic and other properties of the clusters.

One of the important issues is still a resistance of high-temperature superconducting materials, especially nanostructured ones, to various functional and fluctuation effects which primarily affect chemical, thermal, current and magnetic stability and cause energy dissipation in superconductors.

\section{Dissipative phenomena, chemical, thermal, current and magnetic stability of nanostructured high-temperature superconductors}

The problem of functional stability for the composition, structure and properties of superconducting nanostructures under the thermal, current and magnetic influences reflects the problem of the overall nanomaterial 
sustainability in many ways [29]. It depends greatly on the production method: either by nanocluster aggregation, in particular, under conditions close to thermodynamic equilibrium [226,227], or by dispersion from the consolidated substance during non-equilibrium thermodynamic processes while applying outside powerful energy fluxes. The latter method increases greatly the energy saturation of nanoparticles, which may lead to their specific behavior and cause the threshold phenomena (see, for example: [228-234]). In all cases, a significant role in forming the nano-structure stability is played self-organization phenomena [235] including those happening at the formation level of the above-mentioned non-autonomous phases with particular physical chemistry.

Even being outside the functional impacts (while storing or preparing for use), most superconducting compounds are only thermodynamically stable within a limited range of temperatures and oxygen partial pressures, beyond which they are in a state of metastable equilibrium. To stabilize these compounds, dopants are applied that chemically 'deform' the crystal structure and can lead to a significant change in functional parameters of the HTSC material under usage conditions because of geometry changes in superconducting and dielectric areas, as well as the charge redistribution between them. One of the simplest ways to evaluate stability of the synthesized oxide superconductors is based on the known Goldschmidt's geometric tolerance criterion [236,237], according to which the perovskite-like $\mathrm{A}-\mathrm{Cu}-\mathrm{O}$ structure (where $\mathrm{A}$ is for atoms of $\mathrm{K}, \mathrm{Rb}, \mathrm{Cs}, \mathrm{Tl}$, etc.) will be sustainable in the above sense, if the $\left(R_{A}+R_{O}\right) / \sqrt{2}\left(R_{C u}+R_{O}\right)$ ratio lies in the range of $0.8-1$, where $R$ stands for ionic radii of constituent elements. The additional influence of other geometrical factors in the structures of specific chemical compounds is considered, e.g., in $[29,238,239]$. As for the superconducting complex oxides of the last generations representing, as a rule, solid solutions of different non-homogeneity that depends on the production technology and subsequent heat treatment, there is an urgent problem of chemical phase metastability in conditions of low-temperature decomposition of the solid solution supersaturated by one or more components, as well as its instability in relation to small spinodal fluctuations which lead to delamination and formation of coherent areas of significantly different chemical composition [240]. When thermally influenced, nanomaterials may show other processes that affect their stability, particularly those related to the relaxation of internal micro-stresses and the subsequent grain growth, the change in the numerous boundary surfaces and the appropriate proportion of non-autonomous phases in the total material volume, diffusion and effects of border segregation of the individual components $[241,242]$.

These phenomena can become significantly intensified under the influence of various physical fields (magnetic, electric, power, radiation, etc.), primarily functionally conditioned, especially those leading to the above considered thermodynamic phase transitions of a substance or those corresponding to extreme working conditions of type 2 superconducting materials wherein the current density can reach a value of $j=10^{5}-10^{7} \mathrm{~A} / \mathrm{cm}^{2}$, and the superconductivity maintained in magnetic fields is up to $H=10^{5}-10^{6} \mathrm{G}(10-100 \mathrm{~T})$ (Fig. 18, [243]).

Since the superconductors are used due to their ability to resist the damaging effects of high currents and magnetic fields arising at working conditions in most practical applications, much attention is paid to studies of dissipative processes accompanying and contributing to the emergence and evolution of current and magnetic instabilities in superconducting materials. In the development of such instabilities, a special role is played by thermal fluctuations that can lead to overall instability of the critical state itself under certain conditions, which is evidenced by the so-called 'giant' creep phenomenon [244]. As a result, the spontaneous temperature rise taking place in some volume of the superconductor material reduces the pinning forces that bind the individual vortex filaments of the occupied magnetic field to the existing defects, which leads to the vortex lattice disequilibrium and the magnetic flux movement accompanied by heat release due to energy dissipation of the superconducting currents. In turn, this causes a further increase in the sample temperature and, in case of large values of the external magnetic field and transport current in high-temperature superconductors, can turn into a snowballing process of interrelated temperature and electromagnetic field perturbations as a flux jump. In contrast to the usual slow creep of magnetic flux and its logarithmical relaxation through time at small currents, a 'giant creep' can result in the loss of current, magnetic and thermodynamic stability, which ultimately can cause complete destruction of superconductivity $[245,246]$.

A very unusual example of development of these 'thermal' instabilities in superconductors can be the experimentally observed self-similar tree-like or branching formation of normal (non-superconducting) fractal structures which emerge incrementally at temperatures ranging slightly below $T c$ and which are never observed when reaching $T c$. The latter circumstance is still not theory-based despite the large amount of experimental data on such formations which emerge especially well as the macroscopic ones in thin films during the magnetic field penetration (Fig. 19) [247].

A possible explanation of this phenomenon as 'deterministic chaos' [248] can lie in percolation vortex depinning mechanism proposed in [249] to describe the resistive state of thin film of superconductors near the critical current. This approach relates the transition of a superconductor end portion into the resistive state to the viscous 


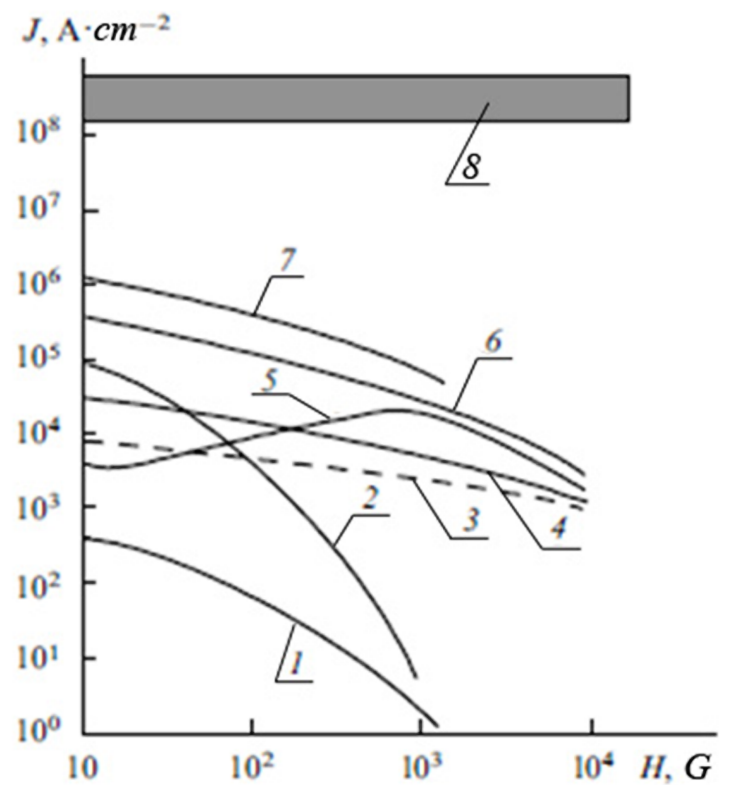

FIG. 18. The stability of the critical-current density for various HTSC materials, depending on the external magnetic field magnitude at $77 \mathrm{~K}$ according to [243]: 1 - sintered ceramics, 2 Bi2212 tapes, 3 - coarse grain ceramics and single crystals, 4 - Y123, 5 - pure Nd123, 6 - Nd123 with ${ }^{235} \mathrm{U}$ embedded and irradiated with slow (thermal) neutrons, 7 - thin films, 8 - the theoretical limit
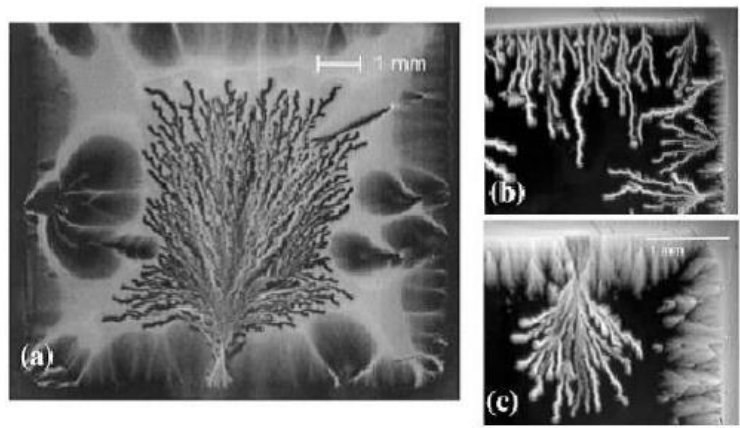

FIG. 19. Magneto-optical images of the treelike branching structures in thin films of $\mathrm{YBaCuO}$ at temperatures of $4.2 \mathrm{~K}(\mathrm{a})$, in $\mathrm{MgB}_{2}$ at a temperature of $3.8 \mathrm{~K}$ (b) and $10 \mathrm{~K}$ (c). Figures taken from the review [247]

flow of vortices and the formation of randomly distributed vortex cores (joined chains of normal state - 'flow clusters'), the probability of formation thereof determines the achievement of the percolation threshold to be below the critical value the total de-pinning (Fig. 20).

This model also results in a universal power law dependence of volt-ampere characteristics of the resistive state of the film superconductors near the critical current and allows us to express the exponent in this dependence through the universal $2 D$ flow theory critical indices of $\gamma \approx 1.3-1.6$ [250]. It agrees satisfactorily with the experimental data for high-temperature superconductors and weakly depends on the HTSC type and sample quality [251] in contrast to conventional type 2 low-temperature superconductors where an exponential dependence of the resistive volt-ampere characteristic is typical.

As noted above, induction ('Meissner') currents that occur near the superconductor surface tend not only to escape the penetration of external magnetic fields in the superconductor but also not to let the vortex filaments of the magnetic field out of its thickness, which penetrated into the sample at temperatures $T>T c$ when it had not been superconductive yet. Therefore, the HTSC stability assessment and the consideration of dissipative 


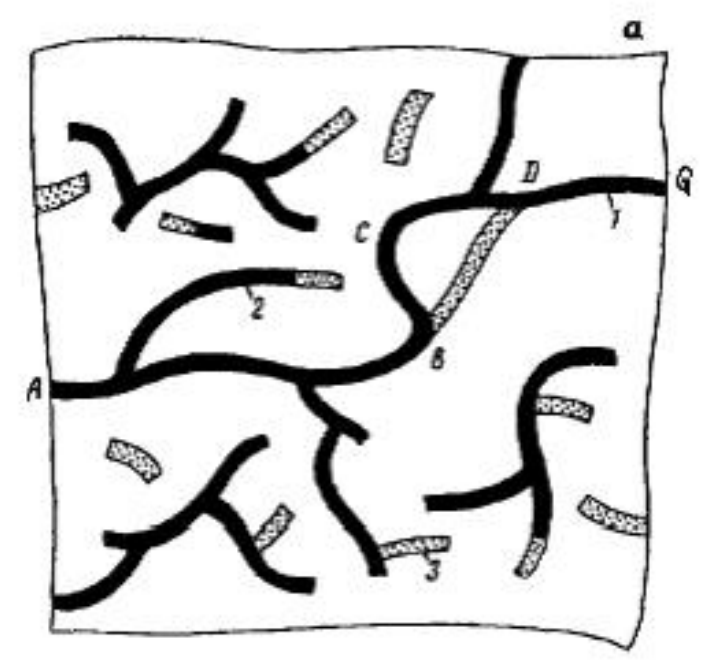

FIG. 20. Percolation 2D-structure in the system of vortex pinning centers in a thin film [249]. Black color denotes the areas where vortices may flow steadily; grey color denotes extra areas appearing when the Lorentz force increases due to the transport current step-up

processes thereof under different perturbations and functional regime regulation should be carried out based on the general non-equilibrium thermodynamics methods [252], taking into account the high-tension electromagnetic field emerging in such materials long before the superconductivity is destructed. With this in mind, we should search the mechanisms to maintain the HTSC superconductivity at the AC current input in overloaded regimes of nonintensively cooled HTS magnets, when the input current peak values can significantly exceed the so-called quench current which defines the boundary of steady states during the DC current flow in the superconductor [38,253].

\section{Conclusion}

The analysis of achievements, challenges and prospects for materials with high transition temperature of the superconducting state indicates a large variety of existing approaches to solve this problem which is relevant in scientific and practical terms.

The obtained experimental results and the proposed theoretical models describing the physical mechanisms of the superconductivity's occurrence at a phenomenological and microscopic level, including those on atom energy spectrum modification, relate the further progress in the understanding of the 'superconducting' gap origin at a temperature below the critical transition, as well as the 'pseudo-gap' above the critical temperature. Although the pseudo-gap origin is not completely understood, it can be considered as an independent phase transition in the substance prior to the transition to a zero-resistance state and insusceptibility to external magnetic field in high temperature superconductors. In this respect, studies of the characteristics of multi-gap and gapless superconducting materials, their abilities to further increase the supercritical transition temperature and the critical current magnitude are also of considerable interest.

Large reserves to create the necessary electron and phonon spectra during the high-temperature superconductivity formation are associated with nanoscale structures and nanoparticle systems of conductors and dielectrics. Herein, a significant role is played by features of their electronic spectrum with a noticeable discrete structure due to the relatively small number of atoms compared to the massive volumes of regular solids, and the formation of the different current contacts between the nanoparticles and the creation of the tunnel circuits. The study of the superconductivity phenomenon at the level of interphase layers (non-autonomous phases) and in the nanoparticles also indicates the possibility to create tunnel chains of metal nanoclusters of certain parameters along with the total charge transfer, where the delocalized electron spectra form energy shells similar to the atomic or nuclear shells. This makes possible the 'vast' intensifying of superconducting pairing and a strong increase in the critical temperature of the superconducting transition.

Implementing of these possibilities requires further study of the superconductivity at the level of the nonautonomous phases in these structures, developing adequate methods of synthesis of nanoparticles of variable 
size, structure and phase composition, as well as consolidation technologies thereof ensuring the preservation of individual nanoparticle superconductivity along with its chemical, thermal, current, and magnetic resistance.

A special role in the instability development, when there are functionally-related effects, is played by thermal fluctuations that are able to lead to the overall supercritical state instability akin to the 'giant' creep phenomenon under certain conditions. It is also necessary to search for the mechanisms to preserve the HTSC superconductivity at $\mathrm{AC}$ current input therein in the overloaded modes when the peak values of input currents can significantly exceed the quench current which sets the boundary of steady states during the DC current flow in the superconductor.

In general, the research lines considered reveal resources to find suitable nanoscale structures and materials with high superconductivity transition temperature.

\section{Post Scriptum}

Before the submission of the present review to the Journal it became known that Nobel prize in Physics 2016 was awarded to David J. Thouless, F. Duncan, M. Haldane and J. Michael Kosterlitz [254] 'for theoretical discoveries of topological phase transitions and topological phases of matter'. The use of the topological concept for studying of space properties which remain unchanged at its remaining invariable at its continuous deformations allows one to describe the order disturbance in the condensed systems, in particular, transition to a superfluid or superconducting state. On this basis the mechanism of phase superconducting transition is explained at low temperatures even in thin (two-dimensional) layers with a stepwise change of conductivity with the increase of induction of the imposed magnetic field as well as the mechanism of disappearance of superconductivity at high temperatures. The latter transition was received the name of Berezinskii-Kosterlitz-Thouless (BKT) transition [255257], but the Soviet physicist-theorist Vadim Berezinskii participated in theory development in the 1970's has not lived up to getting the Nobel Prize. Approximately at the same time F. D. Haldane discovered that topological representations could be used for properties description of the one-dimensional magnetic chains appearing in some materials [258-260]. It is expected that such theoretically grounded search for unusual topological phases (or states) of matter could help researchers in quantum physical phenomena in the field of materials science, electronics, in quantum computer creation.

In this regard it should be also mentioned the work of Yu. F. Antonov and J. B. Danilevich [38] where the topological approach for new type of superconductor topologial electrical machines of high power (the electric engines and electric generators, electromagnetic couplings and DC transformers) has been considered. Here, the local changes of superconductors phase state are considered as topological transformations taking into account that for superconducting structures of any geometrical form and connectivity the fundamental value of quantization of the magnetic flux as the direct consequence of rigid phase coherence remains unchanged as a topological property of those structures.

Technical operation of such machines is provided by electromagnetic effects caused by phase transitions accompanied by the formation of intermediate and mixed states of superconductors and movements of quantum filaments due to the change of connectivity of superconducting circuits using a specially developed resistivesuperconducting switch.

\section{Acknowledgments}

The author would like to thank Prof. V. V. Gusarov for useful discussions. This work was financially supported by the Russian Foundation for Basic Research, project No. 15-08-03238 A.

\section{References}

[1] Physical encyclopedia. Chief editor A.M. Prokhorov. Great Russian encyclopedia, 1998, Moscow, (in Russian).

[2] Kamerlingh-Onnes H. Further experiments with liquid helium. On the electrical resistance of pure metals, etc. VI. On the sudden change in the rate at which the resistance of mercury disappears. Comm. Phys. Lab. Univ. Leiden, 1911, 124.

[3] Meissner W., Ochsenfeld R. Ein neuer Effekt bei Eintritt der supraleitfhigkeit. Naturwiss, 1933, 33 (44), P. $787-788$.

[4] Meissner W. New work on superconductivity. Uspekhi Fizicheskikh Nauk, 1935, 15 (2), P. 208-220 (in Russian).

[5] London F., London H. The Electromagnetic Equations of the Supraconductor. Proc. Roy. Soc. London, 1935, 71, P. 71-88.

[6] The Scientific Letters and Papers of James Clerk Maxwell (1846-1862). Ed. P.M. Harman. Cambridge: University Press, 1990-2002, 1-3.

[7] Ginzburg V.L., Landau, L.D. On the theory of superconductivity. JETP, 1959, 20, P. 1064 (in Russian).

[8] Abrikosov A.A., Gorkov L.P., Dzyaloshinskii I.E. Methods of quantum field theory in statistical physics. Fizmatgiz, Moscow, 1962 (in Russian).

[9] Belyavskii V.I., Kopaev Yu.V. Ginzburg-Landau equations for high-temperature superconductors. Phys. Usp., 2007,50 , P. 540-545.

[10] Bardeen J., Cooper L.N., Schrieffer J.R. Theory of Superconductivity. Phys. Rev., 1957, 108, P. 1175.

[11] Landau L.D., Lifshitz E.M. Curse of Theoretical physics, 9: Statistical physics, Part 2. Nauka, Moscow, 1985 (in Russian). 
[12] Feynman R., Leighton R., Sands M. The Feynman lectures on Physics. Addison-Wesley Publishing Company, Ing, Reading, Massachusetts, Palo Alto. London. 1964, 2.

[13] Fröhlich H. Theory of the superconducting state. Phys. Rev., 1950, 79 (5), P. 845.

[14] Maxwell E. Isotope Effect in the Superconductivity of Mercury. Phys. Rev., 1950, 78, P. 477.

[15] Reynolds C.A., Serin B., Wright W.H., Nesbitt L.B. Superconductivity of isotopes of mercury. Phys. Rev., 1950,78 , P. 487.

[16] Belyavskii V.I., Kopaev Yu.V. First International Conference 'Fundamental Problems of High-Temperature Superconductivity'. Phys. Usp., 2005, 48, P. 177-182.

[17] Fedyukin V.K. The fundamentals of alternative 'theory of superconductivity'. SpbGIEU, St. Petersburg, 2009 (in Russian).

[18] Geballe T.H., Hulm J.K. Bernd Theodor Matthias. National Academies Press: Biographical Memoirs, $1996,70$.

[19] Schrieffer J.R. Theory of Superconductivity. Perseus Books, New York, 1964.

[20] de Genes P.G. Superconductivity of metals and alloys. W.A. Benjamin, New York, 1966.

[21] Lynton E.A. Superconductivity. Methuen \& Co. Ltd, London, 1969.

[22] Ginzburg V.L. The problem of high-temperature superconductivity. II. Sov. Phys. Usp., 1970, 13, P. $335-352$.

[23] Tinkham M. Introduction to superconductivity. McGraw Hil, New York, 1975.

[24] Vonsovskii S.V., Izyumov Yu.A., Kurmaev E.Z. Superconductivity of transition metals, their alloys and compounds. Nauka, Moscow, 1977 (in Russian).

[25] Schmidt V. Itroduction to the physics of superconductors. Nauka, Moscow, 1982 (in Russian).

[26] Abrikosov A.A. The fundamentals of the theory of metals. Nauka, Moscow, 1987 (in Russian).

[27] Ginzburg V.L. On heat transfer (heat conduction) and the thermoelectric effect in the superconducting state. Phys. Usp., 1998, 41, P. 307-311.

[28] Ginzburg V.L. Superconductivity: the day before yesterday - yesterday - today - tomorrow. Phys. Usp., 2000,170 (43), P. $573-583$.

[29] Tretyakov Yu.D., Gudilin E.A. Chemical principles of preparation of metal-oxide superconductors. Russian Chemical Reviews, 2000 , 69 (1), P. 3-40 (in Russian)

[30] Sadovskii M.V. Pseudogap in high-temperature superconductors. Phys. Usp., 2001, 44, P. 515-539.

[31] Ginzburg V.L., Andryushin E.A. Superconductivity. Alpha-M, Moscow, 2006 (in Russian).

[32] Maksimov E.G. High-temperature superconductivity: the current state. Phys. Usp., 2000, 43, P. $965-990$.

[33] Kopaev Yu.V. High-temperature superconductivity models. Phys. Usp., 2002, 45, P. 655-659.

[34] Chernoplekov N.A. State of the art in applied high-current superconductivity. Phys. Usp., 2002, 45, P. 659-665.

[35] Belyavskii V.I, Kopaev Yu.V. Generalizing considerations about the nature of high-temperature superconductivity (based on the proceedings of M2S-HTSC-VII). Phys. Usp., 2004, 47, P. 409-416.

[36] Mourachkine A. Room-Temperature Superconductivity. Cambridge International Science Publishing, Cambridge, 2004.

[37] Rumer Y.B., Ryvkin M.S. Thermodynamics, Statistical Physics and Kinetics. Nauka, Moscow, 1977 (in Russian).

[38] Antonov Yu.F., Danilevich J.B. The Topological Superconducting Electrical Machines. Fizmatlit, Moscow, 2009 (in Russian).

[39] Abrikosov A.A. About the magnetic properties of superconductors of the second group. JETP, 1957, 32, P. 1442 (in Russian).

[40] Abrikosov A.A. The type-II superconductors and the vortex lattice (Materials Nobel Lecture, Stockholm 2003 and Science Division, Argonne National Laboratory, Argonne, USA). Uspekhi Fizicheskikh Nauk, 2004, 174 (11), P. 1235-1239 (in Russian).

[41] Schubnikov L.B., Chotkewitsch B.I., Schepelev J.D., Rjabinin J.N. Magnetische Eigenschaften supraleitender Metalle und Legierungen. Phys. Z. Sowiet, 1936, 10 (2), P. 165-192.

[42] Shubnikov L.V., Khotkevich V.I., Shepelev Yu.D., Ryabinin Yu.N. Magnetic properties of superconducting metals and alloys . JETP, 1937, 7 (2), P. 221-237 (in Russian).

[43] Slezov V.V, Papirov I.I., Shepelev A.G. Discovery of the Shubnikov phase (type ii superconductors). National Science Center, Kharkov Institute of Physics and Technology, 2008, 823 (in Russian).

[44] Tauble H, Essman U. Die Beobachtung magnetischer Strukturen von Supraleitern zweiter Art. Physica Status Solidi, 1967,20 , P. 95-111.

[45] Sarma N.V. Direct evidence for the laminar add flux models of mixed state in type II superconductors. Physics Letters A, 1967, 25 (4), P. 315-316.

[46] San-Zham D., Sarma G., Tomas E. Type II superconductivity. Pergamon press, Oxford-London-Edinburgh-New York Toronto - Sydney - Paris - Braunschweig, 1969.

[47] Zeeman P. Doubles and triplets in the spectrum produced by external magnetic forces. Phil. Mag., 1897, 44, P. 55.

[48] Fulde P., Ferrell R.A. Superconductivity in a Strong Spin-Exchange Field. Phys. Rev. A, 1964, 135, P. 550.

[49] Larkin A.I., Ovchinnikov Yu.N. Inhomogeneous state of superconductors. JETP, 1964, 47 (3), P. $1136-1146$.

[50] Proshin Yu.N., Khusainov M.G. On the manifestations of the state of the Larkin-Ovchinnikov-Fulda-Ferrell in bimetallic structures ferromagnet-superconductor. JETP Letters, 1997, 66 (8), P. 527-532.

[51] Anderson P.W. Theory of dirty superconductors. Journal of Physics and Chemistry of Solids, 1959, 11 (1-2), P. 26-30.

[52] Babaev E., Speight M. Semi-Meissner state and neither type-I nor type-II superconductivity in multicomponent superconductors. Phys. Rev. B, 2005, 72, P. 180502 .

[53] Moshchalkov V., Menghini M., et al. Type-1.5 Superconductivity. Phys. Rev. Lett., 2009, 102, P. 114501.

[54] Vinnikov L.Ya., Karpinski J.,. et al. Vortex structure in $\mathrm{MgB}_{2}$ single crystals observed by the Bitter decoration technique. Phys. Rev. B, 2003, 67, P. 1-3.

[55] Gor'kov L.P., Kopnin N.B. Movement vortexes and resistance of superconductors of the second kind in the magnetic field. Uspekhi Fizicheskikh nauk, 1975, 116 (3), P. 413.

[56] Mints R.G.,. Rakhmanov A.L. Magnetic instabilities in hard orproviding. Uspekhi Fizicheskikh nauk, 1977,121 (3), P. $499-524$ (in Russian).

[57] Malkov M.P., Danilov I.B., Zeldovich A.G. Handbook of physical-technical fundamentals of cryogenics. Energoatomizdat, Moscow, 1985 (in Russian).

[58] Yeshurun Y., Malozemoff A.P. Giant Flux Creep and Irreversibility in an Y-Ba-Cu-O Crystal: An Alternative to the Superconducting-Glass Model. Phys. Rev., 1988, 60, P. 2202-2205.

[59] Buckel W. Superconductivity: Fundamentals and Applications (Physics). Mir, Moscow, 1975 (in Russian). 
[60] Postnikov V.S. Physics and chemistry of solid state. Metallurgy, Moscow, 1978 (in Russian).

[61] URL: http: //elementy.ru/novosti_nauki/431450/Sverkhprovodimost.

[62] Aksenov V.L. Neutron diffraction cuprate high-temperature superconductors. Uspekhi Fizicheskikh Nauk, 2002, 172 (6), P. $701-705$ (in Russian).

[63] Langenberg D.N., Scalapino D.J., Taylor B.N. The Josephson Effects. Scientific American, 1966,214 (5), P. 30.

[64] Langenberg, D.N., Scalapino D.J., Taylor B.N. The Josephson effects. Uspekhi Fizicheskikh Nauk, 1967,91 (2), P. 317-320 (in Russian).

[65] Josephson B.D. Possible new effects in superconductive tunnelling. Phys. Lett., 1962, 1 (7), P. 251-253.

[66] Anderson P.W., Rowell J.M. Probable observation of the Josephson superconducting tunnelling effect. Phys. Rev. Lett., 1963, 10, P. 230-232.

[67] Ponomarev Y.G. Tunnel and St. Andrew spectroscopy of high-temperature superconductors. Uspekhi Fizicheskikh Nauk, 2002, 72 (6), P. 705-711 (in Russian).

[68] Andreev A.F. Thermal conductivity of the intermediate state of superconductors. Journal of Experimental and Theoretical Physics, 1964, 46, P. 18-23 (in Russian).

[69] Andreev A.F. Electrodynamics of the intermediate state of superconductors. Journal of Experimental and Theoretical Physics, 1966, 51, P. 1510 (in Russian).

[70] URL: http://www.nkj.ru/news/24322/.

[71] URL: http://elementy.ru/novosti_nauki/431568/...Yuriy_Erin.

[72] Altshuler T.C, Bresler M.S. On the nature of the energy gap of dodecaborate YbB 12. Physics of the solid state, 2002,44 (8), P. 1465 (in Russian).

[73] Tsidil'kovskii I.M. Gapless semiconductors - a new class of substances. Academy of Sciences of the USSR. Series: Academic reading, Nauka, Moscow, 1986 (in Russian).

[74] Elesin V.F., Kapaev V.V., Kopaev Yu.V. Coexistence of ferromagnetism and nonuniform superconductivity. Phys. Usp., 2004, 47, P. 949953.

[75] Little W.A. Possibility of Synthesizing an Organic Superconductor. Phys. Rev., 1964, 134, P. 416.

[76] Little W.A. Superconductivity at Room Temperature. Scient. Amer, 1965, 212 (2), P. 21.

[77] Little W. Superconductivity at room temperature. Uspekhi Fizicheskikh Nauk, 1965, 86 (2), P. $315-326$ (in Russian).

[78] Bednorz J.G, Muller K.A. Possible highT superconductivity in the Ba-La-Cu-O system. Z. Phys. B: Cond. Mat., 1986,64 (2), P. 189-193.

[79] Tretyakov Yu.D. Chemical superconductors before the Third Millennium. URL: www. chem.msu.su/rus/journals/xr/tretyak.html.

[80] Wu M.K., Ashburn J.R., et al. Superconductivity at $93 \mathrm{~K}$ in a new mixed-phase Y-Ba-Cu-O compound system at ambient pressure. Phys. Rev. Lett., 1987, 58, P. 908.

[81] Maeda H., Tanaka Y., et al. New high-Tc superconductors without rare earth element. Physica C, 1988,153 (602), P. 7.

[82] Matsui Y. Maeda H., Tanaka Y., Horiuchi S. High-resolution electron microscopy of modulated structure in the new high-Tc superconductors of the Bi-Sr-Ca-Cu-O system. Jpn. J. Appl. Phys., 1988, 2 (27), P. 361.

[83] Sheng Z.Z., Hermann A.M. Bulk superconductivity at $120 \mathrm{~K}$ in the Tl-Ca/Ba-Cu-O system. Nature, $1988,332, \mathrm{P} .138-139$.

[84] Torardi C.C., Subramanian M. A., et al. Crystal Structure of $\mathrm{Tl}_{2} \mathrm{Ba}_{2} \mathrm{Ca}_{2} \mathrm{Cu}_{3} \mathrm{O}_{10}$, a 125 K Superconductor. Science, 1988 , 240 (4852), P. 631-634.

[85] Putilin S.N., Antipov E.V., Chmaissem O., Marezig M. Superconductivity at $94 \mathrm{~K}$ in $\mathrm{HgBa}_{2} \mathrm{Cu0} 4+\delta . ~ N a t u r e, 1993,362$, P. $226-228$.

[86] Abakumov A.M., Antipov E.V., et al. Complex oxides with coherent intergrowth structures. Russian Chemical Reviews, 1995, 64 (8), P. 719.

[87] Campbell A.M., Evetts J.E. Critical currents in superconductors. Taylor and Francis Ltd, London, 1972.

[88] Tretyakov Y.D., Gudilin E.A. Chemical design of metal-oxide superconductors. Physica B: Condensed Matter, 2002, 321 (1-4), P. 249256.

[89] Tretyakov Y.D., Gudilin E.A., Reddy E.S., Schmitz G.J. Modern preparation metods of oriented thick films of superconducting cuprates. Crystallography Reports, 2004, 49 (2), P. 233-239.

[90] Reddy E.S., Tarka M., Gudilin E.A., Schmitz G.J. A novel process for textured thick film $\mathrm{YBa}_{2} \mathrm{Cu}_{3} \mathrm{O}_{y}$ coated conductors based on constitutional gradients principle. Superconductor Science and Technology, 2005, 16 (6), P. 859-873.

[91] Martynova I., Tsymbarenko D., et al. Solution deposition of ultrasmooth alumina on long-length metallic substrate for 2G superconducting tapes. Materials Research Bulletin, 2016, 78, P. 64-71.

[92] Liu Zi-Kui, Zhong Yu, Schlom D.G. Computational Thermodynamic Modeling of the Mg-B System. Calpha, 2001,25 (2), P. 299-303.

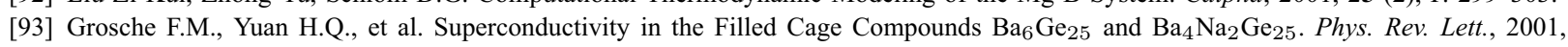
87, P. 107.

[94] Reich S., Tsabba Y. Possible nucleation of a 2D superconducting phase on $\mathrm{WO}_{3}$ single crystals surface doped with Na+. Eur. Phys. J. B, 1999, 9 (1), P. 1-4.

[95] Levi Y., Millo O., et al. Evidence for localized high-Tc superconducting regions on the surface of $\mathrm{Na}^{-\mathrm{doped}} \mathrm{WO}_{3}$. Europhys. Lett., 2000, 51 (5), P. 564-570.

[96] Aliev A.E. High-Tc superconductivity in nanostructured NaxW0 ${ }_{3-y}$ : sol-gel route. Supercond. Sci. Technol., 2008, 21, P. 1-9.

[97] Kamihara Y., Watanabe T., Hirano M., Hosono H. Iron-Based Layered Superconductor La $\left[\mathrm{O}_{1-x} \mathrm{~F}_{x}\right] \mathrm{FeAs}(x=0.05-0.12)$ with $T c=26$ K. Journal of the American Chemical Society, 2008, 130 (11), P. 3296-3297.

[98] Takahashi H., Igawa K., et al. Superconductivity at $43 \mathrm{~K}$ in an iron-based layered compound $\mathrm{LaO}_{1-x} \mathrm{~F}_{x} \mathrm{FeAs}$. Nature, 2008 , 453 (7193), P. 376-378.

[99] Ren Z.A., Lu W., et al. Superconductivity at $55 \mathrm{~K}$ in iron-based F-doped layered quaternary compound $\mathrm{Sm}\left[\mathrm{O}_{1-x} \mathrm{~F}_{x}\right] \mathrm{FeAs}$. Chin. Phys.Lett., 2008, 25, P. 2215.

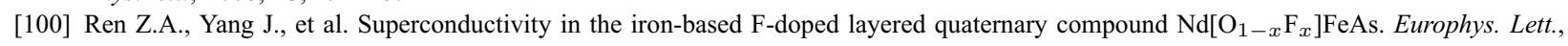
2008, 82 (5), P. 57002.

[101] Wang C., Li L.J., et al. Thorium-doping-induced superconductivity up to $56 \mathrm{~K}$ in $\mathrm{Gd}_{1-x} \mathrm{Th}_{x}$ FeAsO. Europhys. Lett., 2008 , 83 (6), P. 67006. 
[102] Zhao J., Huang Q., Clarina de la Cruz, et al. Structural and magnetic phase diagram of $\mathrm{CeFeAsO}_{1-x} \mathrm{~F}_{x}$ and its relation to hightemperature superconductivity. Nature Materials, 2008, 7, P. 953-959.

[103] Mazin I.I., Singh D.J., Johannes M.D., Du M.H. Unconventional Superconductivity with a Sign Reversal in the Order Parameter of $\mathrm{LaFeAsO}_{1-x} \mathrm{~F}_{x}$. Phys. Rev. Lett., 2008, 101, P. 057003.

[104] Wu M.K., Hsu F.C., et al. The development of the superconducting PbO-type $\beta$-FeSe and related compounds. Physica $C$, 2009, 469 (9-12), P. 340-349.

[105] Guo J., Jin S., et al. Superconductivity in the iron selenide $\mathrm{K}_{x} \mathrm{Fe}_{2} \mathrm{Se}_{2}(0<x<1.0)$. Phys. Rev. B, 2010, 82, P. 180520(1-4).

[106] Martinelli A., Palenzona M., et al. From antiferromagnetism to superconductivity in $\mathrm{Fe}_{1+y} \mathrm{Te}_{1-x} \mathrm{Se}_{x}(0 \leq x \leq 0.20)$ : Neutron powder diffraction analysis A. Martinelli. Phys. Rev. B, 2010, 81, P. 094115(1-10).

[107] Roslova M.V., Lebedev O.I., et al. Diversity of microstructural phenomena in superconducting and nonsuperconducting $\mathrm{Rb}_{x} \mathrm{Fe}_{2}-y \mathrm{Se}_{2}$ : a transmission electron microscopy study at the atomic scale. Inorg. Chem., 2013, 52, P. 14419-14427.

[108] Grinenko V.V., Efremov D.V., et al. Superconducting specific heat jump $\Delta C(T c)$ for $\mathrm{K}_{1-x} \mathrm{Na}_{x} \mathrm{Fe}_{2} \mathrm{As}_{2}$. Physical Review B, 2014, 89, P. 060504(R).

[109] Blinkin A.A., Derevyanko V.V., et al. Evolution of structure and properties of $\mathrm{MgB}_{2}$ superconductor during isothermal annealing. Physics of the solid state, 2005, 47 (9), P. 1546-1551 (in Russian).

[110] Ivanovskii A.L. New superconductors based on five-component transition metal oxypnictides . Uspekhi Khimii, 2010, 79 (1), P. 3-14 (in Russian).

[111] Kopaev Yu.V., Belyavskii V.I., Kapaev V.V. With cuprate luggage to room-temperature superconductivity. Phys. Usp., 2008, 51, P. 191198.

[112] Chen X.K., Konstantinović M.J., et al. Evidence for Two Superconducting Gaps. Phys. Rev. Lett., 2001,87, P. 157002.

[113] Korotkov N.Y., Frolov K.V., et al. Mössbauer Study of a New Superconductor GdOFeAs. Journal of Superconductivity and Novel Magnetism, 2013, 26 (9), P. 2877-?2879.

[114] Moskalenko V.A. Superconductivity of metals taking into account the overlap of the energy bands. Fizika Metals, 1959, 8 (4), P. 503 (in Russian).

[115] Suhl H., Matthias B.T., Walker L.R. Bardeen - Cooper - Schrieffer theory of superconductivity in the case of overlapping bands. Phys. Rev. Lett., 1959, 3, P. 552.

[116] Kuzmicheva T.E., Kuzmichev S.A., et al. Experimental study of intrinsic multiple Andreev reflections effect in GdO(F)FeAs superconductor array junctions. European Physics Letters, 2013, 102, P. 67006.

[117] Lee W.S., Vishik I.M., et al. Abrupt onset of a second energy gap at the superconducting transition of underdoped Bi2212. Nature, 2007, 450 (7166), P. 81.

[118] Mazin I.I., Schmalian J. Pairing Symmetry and Pairing State in Ferropnictides: Theoretical Overview. Physica C, 2009,469 , P. 614-627.

[119] Kuroki K., Onari S., et al. Unconventional Pairing Originating from the Disconnected Fermi Surfaces of Superconducting $\mathrm{LaFeAsO}_{1-x} \mathrm{~F}_{x}$. Phys. Rev. Lett., 2008, 101, P. 087004.

[120] Kontani H., Onari S. Orbital-Fluctuation-Mediated Superconductivity in Iron Pnictides: Analysis of the Five-Orbital Hubbard-Holstein Model. Phys. Rev. Lett., 2010, 104, P. 157001.

[121] Shi-Zeng Lin, Xiao Hu. Vortex States and Phase Diagram of Multi-component Superconductors with Competing Repulsive and Attractive Vortex Interactions. Phys. Rev. B, 2011, 84, P. 214505.

[122] Izyumov Yu.A., Proshin Yu.N., Khusainov M.G. Competition between superconductivity and magnetism in heterostructures of the ferromagnet-superconductor. Successes of physical barriers. Sciences, 2001, 172 (2), P. 113-154.

[123] Wurmeh S., Lebedev O., et al. New Layered Intermetallic Iron-based Superconductors and Related Compounds: Controlling Physical Properties by Using Iso- and Heterovalent Substitutions . Journal RFBR, 2014, 81 (1), P. 64-76 (in Russian).

[124] Kubaschewski O. Iron - Binary Phase Diagrams. Springer-Verlag, Berlin, 1982, 185 p.

[125] Volkov M.P., Melekh B.A.-T., Bakharev V.I., Kartenko N.F. Obtaining poly- and single crystals of superconducting iron monochalcogenides for short times of synthesis. Technical physics Letters, 2010, 36 (6), P. 8-14 (in Russian).

[126] Viewpoint: The iron age of superconductivity. URL: http://physics.aps.org/articles/v1/28.

[127] Chakravarty S., Sudbo A., Anderson P.W., Strong S. Interlayer Tunneling and Gap Anisotropy in High-Temperature Superconductors. Science, 1993, 261, P. 337

[128] Sadovskii M.V. High-temperature superconductivity in layered iron compounds. Uspekhi Fizicheskikh Nauk, 2008,178 (12), P. 12431271.

[129] Leggett A.J. Cuprate Superconductivity: Dependence of Tc on the c-Axis Layering Structure. Physical Review Letters, 1999 , 83 (2), P. 392 (in Russian).

[130] Moler K.A., Kirtley J.R., et al. Images of interlayer Josephson vortices in $\mathrm{Tl}_{2} \mathrm{Ba}_{2} \mathrm{Co}_{6+d}$. Science, 1998, 279 , P. $1193-1196$.

[131] Kotegawa H., Tokunaga Y., et al. Unusual magnetic and superconducting characteristics in multilayered high-Tc cuprates: ${ }^{63} \mathrm{Cu}$ NMR study. Phys. Rev. B, 2001, 64, P. 064515.

[132] Kuzemska I.G., Kuzemsky A.L., Cheglokov A.A. Superconducting Properties of the Family of Mercurocuprates and Role of Layered Structure. Journal of Low Temperature Physics, 2000, 118 (3-4), P. 147-152.

[133] Ginzburg V.L. Once again about high-temperature superconductivity. Contemporary Physics, 1992, 33, P. 15-23.

[134] Drozdov A.P., Eremets M.I., et al. Conventional superconductivity at 203 Kelvin at high pressures in the sulfur hydride system. Nature, 2015, 525, P. 73-76.

[135] Troyan I., Gavriliuk A., et al. Observation of superconductivity in hydrogen sulfide from nuclear resonant scattering. Science, 2016, 351 (6279), P. 1303-1306.

[136] Prins J.F. Ion implantation of diamond for electronic applications. Semiconductor Science and Technology, 2003,8 (3), P. 131.

[137] Scheike T., Böhlmann W., Esquinazi P. et al. Can Doping Graphite Trigger Room Temperature Superconductivity? Evidence for Granular High-Temperature Superconductivity in Water-Treated Graphite Powder. Advanced Materials, 2012, 24 (43), P. $5826-5831$.

[138] Kawashima Y. Possible room temperature superconductivity in conductors. AIP Advanced, 2013, 3 (5), P. 052132.

[139] Tripodi P., Gioacchino D. Di, Borelli R., Vinko J.D. Possibility of high temperature superconducting phases in PdH. Physica C, 2003, 388-389, P. 571-591. 
[140] He R.H., Karapetyan H., et al. From a Single-Band Metal to a High-Temperature Superconductor via Two Thermal Phase Transitions. Science, 2011, 331 (6024), P. 1579-1583.

[141] Damascelli A. Probing the Electronic Structure of Complex Systems by ARPES. Physica Scripta, 2004, 109, P. 61-74.

[142] Stolow A., Bragg A.E., Neumark D.M. Femtosecond time-resolved photoelectron spectroscopy. Chem. Rev., 2004, 104, P. 1719.

[143] Boyarsky L.A. Pseudogap effects in strongly correlated electron systems. Low temperature Physics, 2006,32 (8-9), P. 1078-1084.

[144] Boyarsky L.A. Pseudogap phenomena in superconductors. The gap and the pseudogap in systems with waves of spin/charge-density. The General approach and the applicability of the two-fluid model. Physics of the solid state, semiconductors, nanostructures. Vestnik NSU. Series Physics, 2007, 2, P. 145-151.

[145] Batlogg B., Varma C. The underdoped phase of cuprate superconductors. Phys. World, 2000, 13 (2), P. 33.

[146] Loktev V.M., Quick R.M., Sharapov S.G. Phase actuations and pseudogap phenomena. Not coherent and 'unbalanced' Cooper pairs in HTSC. Phys. Rep., 2001, 349 (1), P. 1-123.

[147] Mathur N.D., Grosche F.M., Julian S.R., et al. Magnetically mediated superconductivity in heavy fermion compounds. Nature, 1998, 394, P. 39.

[148] Izyumov Yu.A. Spin-fluctuation mechanism of high-Tc superconductivity and order-parameter symmetry. Phys. Usp., 1999, 42, P. 215243.

[149] Kaul S.N., Kumar S., Rodriges Fernandez J. et al. Unconventional superconductivity in LaAg1-xMn $\operatorname{Mel}_{x}$ Revance of spin-fluctuationmediated pairing. Europhys. Lett., 2006, 74 (1), P. 138.

[150] Krasnov V.M., Kovalev A.E., Yurgens A., Winkler D. Magnetic field dependence of the superconducting gap and the pseudogap in Bi2212 and HgBr2-Bi2212, studied by intrinsic tunnelling spectroscopy. Phys. Rev. Lett., 2001, 86, P. 2657-2660.

[151] Boyer M.C., Wise W.D., et al. Imaging the two gaps of the high-temperature superconductor $\mathrm{Bi}_{2} \mathrm{Sr}_{2} \mathrm{CuO}_{6}+x \cdot$ Nature Physics, 2007,3 (11), P. 802-806.

[152] Belyavskii V.I., Kopaev Yu.V. Superconductivity of repulsive particles. Phys. Usp., 2006, 49, P. $441-467$.

[153] Aslamazov L.G., Larkin A.I. The Influence of fluctuations on the properties of overproud-nick at temperatures above the critical. Physics of the solid, 1968, 10 (4), P. 1104-1111 (in Russian).

[154] Aslamasov L.G., Larkin A.I. The influence of fluctuation pairing of electrons on the conductivity of normal metal. Phys. Lett. A, 1968, 26, P. 238-239.

[155] Maki K. The Critical Fluctuation of the Order Parameter in Type-II Superconductors. Prog. Theor. Phys., 1968, 39 (4), P. 897-906.

[156] Da Silva Neto E.H., Comin R., et al. Charge ordering in the electron-doped superconductor $\mathrm{Nd}_{2-x} \mathrm{Ce}_{x} \mathrm{CuO}_{4} . \mathrm{Science}_{2} 2015, \mathbf{3 4 7}(6219)$, P. 282-285.

[157] Mizen K.V., Ivanenko O.M. Phase diagram of $\mathrm{La}_{2-x} \mathrm{M}_{x} \mathrm{CuO}_{4}$ as a key to understanding the nature of high temperature superconductors. Uspekhi Fizicheskikh Nauk, 2004, 174 (5), P. 545-563 (in Russian).

[158] Chakravarty S., Kee H.-Y., Vlker K. An explanation for a universality of transition temperatures in families of copper oxide superconductors. Nature, 2004, 428 (6978), P. 53-55.

[159] Aleskovskij V.B. Chemistry and technology of solid substances. J. Appl. Chem. USSR, 1974, 47, P. 2145 (in Russian).

[160] Aleskovski V.B. Chemical Assembly materials. Vestnik Akad. Of Sciences of the USSR, 1975, P. 48-51 (in Russian).

[161] Malygin A.A., Drozd V.E., Malkov A.A., Smirnov V.M. From V.B. Aleskovskii‘s 'Framework' Hypothesis to the Method of Molecular Layering/ Atomic Layer Deposition. Chemical Vapor Deposition, 2015, 21, P. 216-240.

[162] Second-Generation HTS Conductors. Ed. by Goyal A. New York: Inc. Springer-Verlag, 2004.

[163] Tolstoy V.P. Synthesis of thin-layer structures by the ionic layer deposition method. Uspekhi Khimii, 1993, 62 (3), P. $237-242$ (In Russian).

[164] Kuz'menko V.M., Lazarev G.G., Mel'nikov V.I., Sudovcov A.I. Critical parameters of the amorphous metal film (a Review). Ukrainian physical journal, 1976, 21 (6), P. 883-903.

[165] Eletskii A.V., Smirnov B.M. Fullerenes and carbon structures. Phys. Usp., 1995, 38, P. $935-964$ (in Russian).

[166] Galikeev A.R. New forms of polymeric hydrocarbon. Ufa: Ufa. State oil. Tech. Univ., 2001.

[167] Yang H.B., Rameau J.D., Johnson P.D., at al. Emergence of preformed Cooper pairs from the doped Mott insulating state in

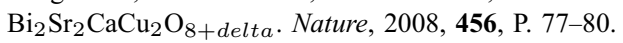

[168] Shabanova N.P., Krasnoslobodtsev S.I., Varlashkin A.V., Golovashkin A.I. Parallel critical magnetic field of thin films NbC and MgB ${ }_{2}$ Scientific session Moscow engineering physics Institute, 2006, 4.

[169] Bagraev N.T., Klyachkin L.E., et al. Superconducting properties for silicon nanostructures. Semiconductor physics and technology, 2009, 43 (11), P. 1481-1495 (in Russian).

[170] Prikhod'ko A.V., Kozyrev S.V. Superconductivity in quantum-well nanostructures. Scientific and technical sheets SPbSPU, 2007, 4, P. 221-226 (in Russian).

[171] Shapoval E.A. Critical Fields of Thin Superconducting Films. JETP, 1966, 22 (3), P. 647-653 (in Russian).

[172] Pearl J. Current distribution in superconducting films carrying quantized fluxoids. Appl. Phys. Lett., 1964,5 , P. 65.

[173] Samus A.N., Popkov A.F., et al. Resistive State and dynamics of vortices in superconducting films. Superconductivity: physics, chemistry, technology, 1991, 4 (7), P. 1324 (in Russian).

[174] Lomtev A.I. Contrails live an isolated vortex pearl in a thin film magnetic superconductor. JETP Letters, 2000 , 71 (10), P. 618 (in Russian).

[175] Lomtev A.I. Inversion satellites" secluded oscillating vortex pearl in a thin film magnetic superconductor. Physics of the solid state, 2001, 43 (11), P. 1945-1951 (in Russian).

[176] Umezawa H., Matsumoto H., Tachiki M. Thermo Field Dynamics and Condensed States. Amsterdam (Netherlands): North-Holland Publishing Company, 1982.

[177] Grishin A.M., Martynovich A.Y., Yampolsky S.V. Inversion of the magnetic field and the chain of vortices in anisotropic superconductors. JETP, 1990, 97 (6), P. 1930-1946 (in Russian).

[178] Buzdin A.I., Simonov A.Yu. Magnetic flux Penetration in layered superconductors. JETP, 1990, 98 (6), P. $2074-2085$ (in Russian).

[179] Kaul S.N., Kumar S., Rodriges Fernandez J. et al. Unconventional superconductivity in $\operatorname{LaAg}_{1-x} \mathrm{Mn}_{x}$ : Relevance of spin-fluctuationmediated pairing. Europhys. Lett., 2006, 74 (1), P. 138. 
[180] Ipatova I.P., Shchukin V.A., et al. Formation of strained superlattices with a macroscopic period via spinodal decomposition of III-V semiconductor alloys. Sol. St. Commun., 1991, 78 (19), P. 19-24.

[181] Bagraev N.T., Gehlhoff W., et al. Quantum-Well Boron and Phosphorus Diffusion Profiles in Silicon. Def. Dif. Forum, 1997, 143-147, P. 1003.

[182] Malygin A.A. Nanotechnology molecular layering: principles and applications (a review). Nanotechnologies in Russia, 2007, 2 (3-4), P. 87 (in Russian).

[183] Mazel E.Z., Press F.P. Planar silicon technology devices. Moscow, Energy, 1974 (in Russian).

[184] VLSI Technology: Fundamentals and Applications Editors. Ed. by Yasuo Tarui. Springer Series in Electronics and Photonics, 12, Springer-Verlag, 1986.

[185] Jaeger R.C. Film Deposition - Introduction to Microelectronic Fabrication (2nd). UpPer Saddle River: Prentice Hall, 2002

[186] Poindexter E.H., Caplan P.H., Deal B.E., Gerardy G.J. The Physics and Chemistry of $\mathrm{SiO}_{2}$ and $\mathrm{Si}_{-} \mathrm{SiO} \mathrm{O}_{2}$ Interfaces. Plenum Press, New York, 1988.

[187] Bagraev N., Bouravleuv A., et al. Self-assembled impurity superlattices and microcavities in silicon. Def. Dif. Forum, 2001 , 194 , P. 673.

[188] Feder J. Fractals. Plenum Press, New York, 1988.

[189] Tsebro V.I., Omeljnovskij O.E., Moravian A.P. Undamped currents and capture the magnetic flux in the fragments of the carbon deposits containing multilayer nanotubes. JETP Letters, 1999, 70 (7), P. 457-462 (in Russian).

[190] Khusainov M.G. Nonuform superconducting states and umklapp processes in ferromagnet-superconductor nanostructuctures. E. J. Magnetic Resonance in Solid, 2004, 6 (1), P. 104-118.

[191] Morozov Y.G., Belousova O.V., et al. Electric field-assisted levitation-jet aerosol synthesis of Ni/NiO nanoparticles. Journal of Materials Chemistry, 2012, 22 (22), P. 11214-11223.

[192] Kuznetsov M.V., Morozov Y.G., Belousova O.V. Levitation jet synthesis of nickel ferrite nanoparticles. Inorganic Materials, 2012, 48 (10), P. 1044-1051.

[193] Morozov Y.G., Ortega D., et al. Some peculiarities in the magnetic behavior of aerosol generated NiO nanoparticles. Journal of Alloys and Compounds, 2013, 572, P. 150-157.

[194] Shishkovsky I., Morozov Y. Electrical and magnetic properties of multilayer polymer structures with nano inclusions as prepared by selective laser sintering. Journal of Nanoscience and Nanotechnology, 2013, 13 (2), P. 1440-1443.

[195] Gusarov V.V., Malkov A.A., Ishutina Zh., Malygin A.A. Phase formation in a nanosize silicon oxide film on surface of aluminum oxide. Tech. Phys. Lett., 1998, 24 (1), P. 3-8 (in Russian).

[196] Smirnov A.V., Fedorov B.A., et al. Core-shell nanoparticles forming in the $\mathrm{ZrO}_{2}-\mathrm{Gd}_{2} \mathrm{O}_{3}-\mathrm{H}_{2} \mathrm{O}$ system under hydrothermal condition. Doklady Physical Chemistry, 2014, 456 (1), P. 171-173 (in Russian).

[197] Almjasheva O.V., Smirnov A.V., et al. Structural features of $\mathrm{ZrO}_{2}-\mathrm{Y}_{2} \mathrm{O}_{3}$ and $\mathrm{Gd}_{2} \mathrm{O}_{3}$ nanoparticles forming under hydrothermal condition. Russian Journal of General Chemistry, 2014, 84 (5), P. 710-716 (in Russian).

[198] Tomkovich M.V., Andrievskaya E.R., Gusarov V.V. Formation under hydrothermal condition of nanoparticles based on system ZrO ${ }_{2}-$ $\mathrm{Gd}_{2} \mathrm{O}_{3}$. Nanosystems: Physics, Chemistry, Mathematics, 2011, 2 (2), P. 6-14 (in Russian).

[199] Gusarov V.V., Almjasheva O.V. The role or status of substances in the formation of structure and properties of materials. Chapter 13 in the book Nanomaterials: properties and applications. Ed. by Yaroslavtsev A.B. Scientific world, Moscow, 2014 (in Russian).

[200] Defay R. Etude Thermodynamique de la Tension Superficielle. Paris, Gauthier-Villars and Cie, 1934,372 p.

[201] Defay R., Prigogine I. Tension superficielle et adsorption. Liege, Ed. Desoer, 1951, 295 p.

[202] Gusarov V.V., Suvorov A.S. Self-accelerating processes of development of solid-phase systems (synthesis and function). In book Future directions of chemistry and chemical technology. Ed. S. Dudyrev. Chemistry, Leningrad, 1991, P. 153-158 (in Russian).

[203] Gusarov V.V., Ishutin J.N., Malkov A.A., Malygin A.A. Peculiarities of solid-phase chemical reaction of mullite formation in nanoscale film composition,. Dokl. Academy of Sciences, 1997, 357 (2), P. 203-205 (in Russian).

[204] Tauson, V.L., Loginov B.A., Akimov V.V., Lipko V.S. Sticky Nonautonomous phases as potential sources of incoherent elements. Dokl. Academy of Sciences, 2006, 406 (6), P. 806 (in Russian).

[205] Neiman A.Ya., Uvarov N.F., Pestereva N.N.. Solid state surface and interface spreading: An experimental study. Solid State Ionics, 2007, 177 (39-40), P. 3361.

[206] Rusanov A.I. Phase equilibria and surface phenomena. Chemistry, Leningrad, 1967, 388 p. (in Russian).

[207] Pervov V.S., Mikheikin I.D., Makhonina E.V., Buckiy V.D. Supramolecular assemblies in eutectic alloys. Uspekhi Khimii (Progress in chemistry), 2003, 72 (9), P. 852 (in Russian).

[208] Neumann A.J., Tsipis E.V., et al. Surface. X-ray, synchrotron and neutron studies, 2001, 10, P. 68 (in Russian).

[209] Kafarov V.V., Meshalkin V.P. Analysis and synthesis of chemical-technological systems. Chemistry, Moscow, 1991,432 p. (in Russian).

[210] Cohen M.H., Douglass D.H. Superconductive Pairing Across Electron Barriers. Phys. Rev. Lett., 1967,19 , P. 118.

[211] Keldysh L.V. Superconductivity in nonmetallic systems. Sov. Phys. Usp., 1965, P. 496-500 (in Russian).

[212] Morozov Y.G., Petinov V.I. Superconductivity in the ensembles of small metallic particles. Solid State Communs., 1981,40 , P. 991.

[213] Geppert-Mayer M., Jensen J.H.D. Elementary Theory of Nuclear Shell Structure. John Wiley u. Sons, Inc., New York; Chapman and Hall, Ltd., London, 1955.

[214] Goeppert Mayer M. Nuclear Shell Structure. Modern Physics for the Engineer, McGraw Hill, 1961.

[215] Goeppert Mayer M. Nuclear Shell Structure. Uspekhi Fizicheskikh Nauk, 1964, 82 (4), P. 749-768 (in Russian).

[216] Trunev A.P. The structure of atomic nuclei and binding energy in the shell model. J. Chaos and Correlation, 2012, 1, P. 1-19.

[217] Friedel J. BCS superconductivity for weakly coupled clusters. Journal de Physique II, EDP Sciences, 1992, 2 (4), P. 959-970.

[218] Kresin V.Z., Ovchinnikov Yu.N. 'Giant' strengthening of superconducting pairing in metallic nanoclusters: strong increase in the transition temperature and the possibility of superconductivity at room temperature. Uspekhi Fizicheskikh Nauk, 2008, 78 (5), P. 449 (in Russian).

[219] Perenboom J., Wyder P., Meier F. Electronic-properties of small metallic particles. Phys. Rep., 1981, 78, P. 173-292.

[220] Anderson P.W. Theory of dirty superconductors. Journal of Physics and Chemistry of Solids, 1959, 11 (26), P. 26-30.

[221] Abeles B. Applied Solid State Science. In Advances in Material and Device Research, ed. by R. Wolfe. Academic Press, New York, 1976, 6, P. 1.

[222] Dynes R.C., Garno J.P., Rowell J.M. Electrical Conductivity in Quench-Condensed Metal Films. Phys. Rev. Lett., 1978, 40, P. 479. 
[223] Deutscher G. New Superconductors: From Granular to High Tc. World Scientific, Singapore, 2006,244 p.

[224] Parmenter, R. Size Effect in a Granular Superconductor. Phys. Rev., 1968, 166, P. 392.

[225] Knight W. In Novel Superconductivity, Ed. by S.A. Wolf, V.Z. Kresin. New York: Plenum Press, 1987.

[226] Pozhidaeva O.V., Korytkova E.N., Drozdova I.A., Gusarov V.V. Phase state and particle size of ultradispersed zirconium dioxide as influenced by conditions of hydrothermal synthesis. Russian Journal of General Chemistry, 1999, 69 (8), P. 1219-1222.

[227] Almyasheva O.V., Gusarov V.V. Features of the phase formation in the nanocomposites. Russian Journal of General Chemistry, 2010, 80 (3), P. 385-390.

[228] Uvarov N.f., Boldyrev V.V. Dimensional effects in chemistry of heterogeneous systems. Progress of chemistry, 2001 , 70 (4), P. $307-329$.

[229] Mastai Y., Gedanken A. In The Chemistry of Nanomaterials: Synthesis, Properties and Applications, Ed. by Rao C.N.R., Muller A., Cheetham A.K. Weinheim: Wiley-VCH, 2004, 1, P. 113.

[230] Gusarov V.V. The thermal effect of melting in polycrystalline systems. Thermochim. Acta, 1995, 256 (2), P. $467-472$.

[231] Kovalenko A.N., Kalinin N.V. Thermodynamic instability of Compound and for-mation of nanosized parti-cles nearby the critical point of phase generating media. Nanosystems: physics, chemistry, mathematics, 2014, 5 (2), P. 258-293.

[232] Bulgakov A.V., Bulgakova N.M. Burakov I.M., etc. The Synthesis of nanoscale materials under the influence of powerful streams of energy on matter. Institute of Thermophysics SB RAS, Novosibirsk, 2009.

[233] Kotov Yu.A., Bagazeev A.V., Beketov I.V. etc. Characteristics of nickel oxide nanopowder obtained an electric explosion delay. Technical physics letters, 2005, 75 (10), P. 39-43 (in Russian).

[234] Sedoy V.S., Valevich V.V., Gerasimova N.N. Synthesis of highly dispersed powders method of electric explosion in Gaza with a pressure. Physics and chemistry of materials processing FHOM, 1999, 4, P. 92-95 (in Russian).

[235] Tretyakov Yu.D. Self-organisation processes in the material chemistry. Uspekhi Khimii (Russian Chemical Reviews), 2003, 72 (8), P. 731-763.

[236] Goldschmidt V.M. Die Gesetze der Krystallochemie. Die Naturwissenschaften, 1926, 21, P. $477-485$.

[237] Urusov V.S., Eremin N.N. Crystal chemistry. Publishing house of Moscow University, Moscow, 2005 (in Russian).

[238] Tugova E.A. A comparative analysis of the formation processes of Ruddlesden-Popper phases in the $\mathrm{La}_{2} \mathrm{O}_{3}-\mathrm{SrO}_{-} \mathrm{M}_{2} \mathrm{O}_{3}(\mathrm{M}=\mathrm{Al}, \mathrm{Fe})$ systems. Glass Physics and Chemistry, 2009, 35 (4), P. 416-422.

[239] Tugova E.A., Gusarov V.V. Influence of structural mismatch of the layers forming the phase Ruddlesden-Popper $\mathrm{Ln}_{n} \mathrm{MFe}_{n} \mathrm{O}_{3 n+1}$ on their resistance. Proceedings of VIII International conference 'Amorphous and microcrystalline semiconductors'. St. Petersburg, 2012, p. 329-330.

[240] Goodilin E.A., Oleynikov N.N., et al. On the stability region and structure of the $\mathrm{Nd}_{1+x} \mathrm{Ba}_{2-x} \mathrm{Cu}_{3} \mathrm{O}_{z}$ solid solution. Physica $C$, 1996 , 272 (182), P. 65-78.

[241] Andrievskii R.A. Thermal stability of nanomaterials. Uspekhi khimii (Russian Chemical Reviews), 2002,10 (71), P. 853-866.

[242] Andrievskii R.A. Thermal stability of consolidate metallic nanomaterials. Uspekhi khimii (Russian Chemical Reviews), 2014, 4, P. 365375.

[243] Mints R.G., Rakhmanov L.L. Magnetic instabilities in hard superconductors. Uspekhi Fizicheskikh Nauk, 1977,121 (3), P. 499-524 (in Russian).

[244] Romanovskii V.R. Macroscopic flux-creep electrodynamics of high temperature superconductor. In book Studies of high temperature superconductors. Advances in research and applications. Nova Science Publishers, New York, 2005.

[245] Romanovskii V.R. Thermal mechanisms of the formation, destruction, and degradation of critical current states of high-temperature superconductors. Doklady Physics, 2009, 54 (4), P. 196.

[246] Romanovskii V.R. Magnetic, overcurrent and thermal instabilities in superconductors (review results of existing stability theory). Vestnik Bauman Moscow State Technical University, Ser. 'Engineering', 2012, P. 17-32 (in Russian).

[247] Altshuler E., Johansen T.H. Colloquium: Experiments in vortex avalanches. Rev. Mod. Phys., 2004,76, P. 471.

[248] Schuster H.G. Deterministic Chaos - An Introduction Physik. Verlag, Weinheim, 1984.

[249] Snarsky A.A., Palicky E.A., Palti A.M., Morozovskii A.E. Percolation mechanism of depinning vortices in the resistive state of thin films of superconductors of the second kind. JETP Letters, 1995, 61 (2), P. 112-116 (in Russian).

[250] Shklovsky B.I., Efros A.L. Percolation Theory and conductivity of strongly inhomogeneous media. Uspekhi Fizicheskikh Nauk, 1975, 117 (3), P. 401-433 (in Russian).

[251] Solovjev V.A., Pan V.M., Freyhard H.C. Anisotropic flux dynamics in single-crystalline and melt-textured $\mathrm{YBa}_{2} \mathrm{Cu}_{3} \mathrm{O}_{7}$ ? $\delta$. Phys. Rev. B, 1994, 50 (18), P. 13724-?13734.

[252] Kovalenko A.N. Regulation and thermodynamic stability of nonequilibrium processes of rapid transformation of energy. Proceedings of CKTI (I.I.Polzunov Joint Stock Company CKTI), 1996, 281 (2), P. 53-62 (in Russian).

[253] Arkharov A.M., Lavrov N.A., Romanovskii V.R. Features conditions electrodynamic stabilization of composites based on hightemperature superconductors with different types of nonlinearity in their current-voltage characteristics. Journal of technical physics, 2014, 84 (6), P. 77-85 (in Russian).

[254] URL: https://www.nobelprize.org/nobel_prizes/physics/laureates/2016/press.html.

[255] Hadzibabic Z., Krüger P., et al. Berezinskii-Kosterlitz-Thouless crossover in a trapped atomic gas. Nature, 2006, 441, P. 1118.

[256] Berezinskii V.L. Destruction of long-range order in one-dimensional and two-dimensional systems having a continuous symmetry group I. Classical systems. Sov. Phys. JETP, 1971, 32 (3), P. 493-500.

[257] Kosterlitz J.M., Thouless D.J. Ordering, metastability and phase transitions in two-dimensional systems. Journal of Physics C: Solid State Physics, 1973, 6, P. 1181-11203.

[258] Gibney E., Castelvecchi D. Physics of 2D exotic matter wins Nobel: British-born theorists recognized for work on topological phases. Nature. London: Springer Nature, 2016, 538 (7623), P. 18

[259] Haldane F.D.M. Quantum Hall effect without Landau levels: a condensed-matter realization of the parity anomaly. Phys. Rev. Lett., 1988, 61, P. 2015.

[260] Haldane F.D.M. Geometrical Description of the Fractional Quantum Hall Effect. URL: https://arxiv. org/abs/1106.3375, 2011. 